\title{
GEOGRAFÍA CULTURAL DE LOS ARCHIPIÉLAGOS DE FUEGO-PATAGONIA EN TIEMPOS RECIENTES. MOVILIDAD Y PLANIFICACIÓN
}

\author{
LUIS ALBERTO BORRERO ${ }^{a}$, FLAVIA MORELLO ${ }^{b} \&$ MANUEL SAN ROMÁN ${ }^{b}$
}

\begin{abstract}
RESUMEN
La sistematización del concepto de geografía cultural y su utilidad para jerarquizar el espacio de acuerdo a valores y conocimientos culturales que disponen de visibilidad y obstrusividad arqueológica se investigan en consideración a las dinámicas de movilidad y circulación humana en los paisajes fueguinos del meridión, buscando evaluar su grado de variación ante distintos factores y circunstancias. El trabajo se propone trascender los compartimientos étnicos reconocidos por las observaciones históricas y los estudios etnográficos, se focaliza en tiempos recientes $y$, también, se integra con distintos enfoques y conceptos especializados del registro arqueológico de estudios de sistemas tecno-económicos y la arqueozoología, entre otros.
\end{abstract}

PALABRAS CLAVE: fueguino, Patagonia, arqueología, adaptación, movilidad, planificación, organización cazadores-recolectores.

\section{CULTURAL GEOGRAPHY OF THE ARCHIPELAGOS OF FUEGO-PATAGONIA DURING RECENT TIMES. MOBILITY AND PLANNING}

\begin{abstract}
The systematization of the concept of cultural geography and its usefulness in hierarchizing space according to cultural values and knowledge that have archaeological visibility and obstrusiveness, are reviewed. They consider the dynamics of mobility and human circulation in the Fuegian landscapes of the austral region, evaluating its variation under different conditions. The work aims to transcend the ethnic boundaries recognized by historical observations and ethnographic studies, focuses on recent times and, also, it is integrated with different approaches and concepts of specialized fields within the archaeological record, archaeozoology or studies of techno-economic systems, among others.

a Universidad de Buenos Aires e Instituto Multidisciplinario de Historia y Ciencias Humanas (IMHICIHU-CONICET), Saavedra 15, piso $5^{\circ}$ (1083 ACA), Ciudad Autónoma de Buenos Aires, Argentina. $\$ laborrero2003@yahoo.com

b Centro de Estudios del Hombre Austral, Instituto de la Patagonia, Universidad de Magallanes, Av. Bulnes 01890, Punta Arenas, Chile.flavia.morello@umag.cl manuel.sanroman@umag.cl
\end{abstract}


KEY WORDS: Fuegian, Patagonia, archaeology, adaptation, mobility, planning, hunter-gatherers organization.

\section{INTRODUCCIÓN}

El foco de nuestra investigación se concentra en las problemáticas que abarca la escala de las adaptaciones fueguinas meridionales para aproximadamente los últimos 1.000 años en el sur de Fuego-Patagonia. Las características generales de estas adaptaciones ya han sido bien establecidas en una escala regional (Legoupil, 1985-1986; Orquera \& Piana, 1999a; San Román, 2014). Son abundantes los estudios arqueológicos en isla Dawson, el canal Beagle y se están multiplicando los trabajos en la península Mitre, pero continúan siendo muy escasas las contribuciones del sur y el occidente del archipiélago fueguino. La información etnográfica también presenta esta disparidad. A pesar de nuestro foco en los tiempos más recientes, el trabajo busca trascender los compartimientos étnicos reconocidos por las observaciones históricas y los estudios etnográficos (Santiago \& Vázquez, 2009; Borrero et al. 2011; Morello, 2016; entre otros). Muy apropiadamente Emperaire destacó la dificultad de asignar etnicidad a los restos arqueológicos de las islas meridionales (Emperaire, 1963 [1955], p. 65), problema que fácilmente puede extenderse a toda la región y que constituye una fuerte razón para no forzar las analogías haush, yagán o kawésqar ante los datos arqueológicos, por recientes que éstos sean. Esto significa que dicho acercamiento metodológico no nos parece adecuado para los objetivos de este trabajo. Obviamente, no se omitirá la atribución étnica reconocida por la mayoría de las observaciones de viajeros y etnógrafos, pero nos parece útil no centralizar el análisis en esa atribución. Esto evita varios problemas que resultan de tratar de asimilar informaciones únicas, que chocan contra la generalidad de las observaciones, muchas de ellas provenientes de zonas de disputadas y dinámicas atribuciones étnicas. Por otra parte, no pensamos que el hallazgo de una anomalía en la literatura etnográfica, o sea una observación que difiere de todas las demás, sirva para dudar de la observación original (Borrero, 1984; Terradas et al. 1999). No solamente no hay base epistemológica para sostener que algo no ocurrió simplemente porque sólo se lo registró una vez, sino que puede tratarse de situaciones creadas por circunstancias excepcionales o simplemente resultar de las decisiones de un individuo que puede desafiar la norma (Borrero, 1991). Más pertinente para nuestro trabajo es considerar que en algunos casos puede tratarse de confusiones creadas por la atribución étnica. Todas estas son discusiones necesarias, pero no nos ocuparemos de ellas en esta ocasión. Asimismo, no presentamos aquí análisis exhaustivos de las referencias etnográficas, tema para el que existen excelentes fuentes (Cooper, 1917; Orquera \& Piana, 1999b, 2015). El objetivo del trabajo es el estudio de la movilidad humana en el archipiélago fueguino en general, aspirando a que esto constituya una base sobre la cual estudiar en el futuro aspectos puntuales, tales como la circulación de bienes, el uso del hinterland (espacios interiores) y la importancia del equipamiento en el uso del espacio.

\section{GEOGRAFÍA CULTURAL}

El concepto de geografía cultural refiere al uso de un ambiente y recursos dentro del sistema de conocimientos disponibles, en el que humanos y ambiente coevolucionan (Binford, 1987). Responde a la sutil interacción entre seres humanos, otros organismos y ambientes en variables escalas y se refiere a "un estado de conocimiento expresado en el espacio sobre la base del cual se organiza la instalación y circulación humana del paisaje. Se trata, por ello, de una entidad de alcance cambiante" (Borrero \& Borrazzo, 2011, p. 7). Entonces, alude a un espacio organizado, con lugares residenciales, de adquisición de recursos, de usos múltiples, de tránsito o de evitación. En una lectura estricta, el concepto de geografía cultural sirve para jerarquizar el espacio de acuerdo con valores culturales que disponen de visibilidad y obstrusividad arqueológica. El concepto está relacionado con el de "site territory", tal como lo presentaron Higgs y Vita-Finzi (1972, p. 30, ver Binford, 1982), e incluye muchos de los elementos recientemente integrados dentro de las líneas de investigación de 
construcción de nicho (Odling-Smee et al. 2003). En términos del estudio del registro arqueológico ese concepto es coordinable con distintos enfoques arqueozoológicos, antracológicos o de estudios de sistemas tecno-económicos (cfr. Lemonnier, 1983, 1991; Inizan et al. 1995; Álvarez et al. 2010; Enloe, 2010; Borrero \& Borrazzo, 2011; Caruso Fermé et al. 2011; de Beaune, 2011; Borrazzo, 2012; Christensen, 2016; Morello, 2016).

El trabajo se concentra en la región ubicada al sur del estrecho de Magallanes occidental y el meridión de la expresión fueguina de la cordillera de los Andes e incluyendo tanto la cordillera Darwin como su extensión, de bastante menor altura hacia el este. Los archipiélagos del sur presentan mucha variación ambiental, que se puede medir principalmente a través de las isoyetas, que mínimamente varían de ca. 500 $\mathrm{mm}$ anuales al este a más de $3.000 \mathrm{~mm}$ anuales al oeste (Venegas \& Sielfeld, 1979; Zamora \& Santana, 1979; Tuhkanen et al. 1989-1990; Arroyo et al. 1996). Dentro de ese continuum pluviométrico la geografía isleña meridional se puede segmentar mínimamente en sectores centro-oriental y sudoccidental, con el punto de inflexión ubicado un poco al oeste de Yendegaia y una separación graduada a partir de la isoyeta de $1.000 \mathrm{~mm}$ anuales. Esto ubica por la franja centroeste al canal Beagle, la costa de la península Mitre y las islas orientales como de los Estados, Picton y Lennox, mientras que por el sudoeste primero se bordea la península Brecknock y luego se ubican las islas del borde del Pacífico, como Covadonga o Desolación, las costas sudoccidentales de isla Hoste y las cadenas de islas que llegan al cabo de Hornos, entre otros (Fig. 1).

La introducción de registros etnohistóricos previos al siglo XIX indica cambios climáticos importantes a través del tiempo. Se ha registrado hielo flotante en el estrecho de Magallanes durante el siglo XVI (Prieto \& Herrera, 1999), así como los estudios paleoambientales identificaron variaciones en la productividad en el sector central del mismo (Aracena et al. 2015).

El ambiente de todo el sector sudoeste tiene un clima riguroso, al que se suman las tormentas y el oleaje del océano Pacífico (Caniupán et al. 2014). El segmento occidental del canal Beagle es muy húmedo, presenta bosque tupido y costas abruptas y glaciares que llegan a la costa y desprenden témpanos de rápida desintegración (Promis et al. 2008; Coronato et al. 2017). Los glaciares de la cara norte de la cordillera Darwin han retrocedido mucho durante los últimos años, por ejemplo, el glaciar Marinelli retrocedió unos 15 km entre 1913 y 2011 (Bown et al. 2014), al punto de no producir témpanos con la misma intensidad en los fiordos del seno Almirantazgo con respecto a lo que ocurre en el frente sur de la cordillera Darwin. Estos cambios han redundado en diferente disponibilidad de espacio, fluctuaciones en la salinidad del agua (lo que afecta su productividad), creación de renoval y otros aspectos sensibles para la habitación humana (Andrade, 1991; Cárdenas \& Montiel, 2016; Försterra et al. 2016). El segmento central del canal Beagle que va desde Yendegaia a Almanza en la costa norte y la costa de Navarino al sur es considerado el más adecuado para la instalación humana (Orquera \& Piana, 2015, p. 33), lo que tiene fuerte respaldo arqueológico (Orquera \& Piana, 1999a; Ocampo \& Rivas, 2000; Zangrando et al. 2014, 2016; Mardones, 2016, 2019). Presenta costas protegidas, sin presencia de hielo. El segmento oriental del canal, a partir de Almanza hacia el este, incluye la isla Gable, además de otras islas e islotes. Por supuesto hay heterogeneidad en cada una de estas unidades, aunque Orquera y Piana (2015, p. 71) aclaran que desde el Beagle a Cabo de Hornos "no debe ser considerado un ambiente en mosaico". Sin embargo, el contraste entre homogeneidad y heterogeneidad es escalar, pues en alguna escala todos los ambientes son heterogéneos (Jochim, 1981, p. 49). La distinción de tres ecozonas en la región del canal Beagle destaca algunas de estas importantes variaciones (Tivoli \& Zangrando, 2011).

Los ambientes más abundantes son los de tierras húmedas, particularmente turberasybosques. En el este predominan las turberas esfagnosas y de Carex, que se cuentan entre las más orientales de América del Sur (Arroyo et al. 2005). En términos generales se reconocen variadas formas de bosque a lo largo del Beagle, incluyendo el magallánico perennifolio y el magallánico deciduo, en tanto que hacia el oeste lo prevaleciente son turbales, matorrales y árboles achaparrados, incluyendo sectores de krummholz (Moore, 1983; Tuhkanen et al. 1989-1990; Promis et al. 2008; Orquera 


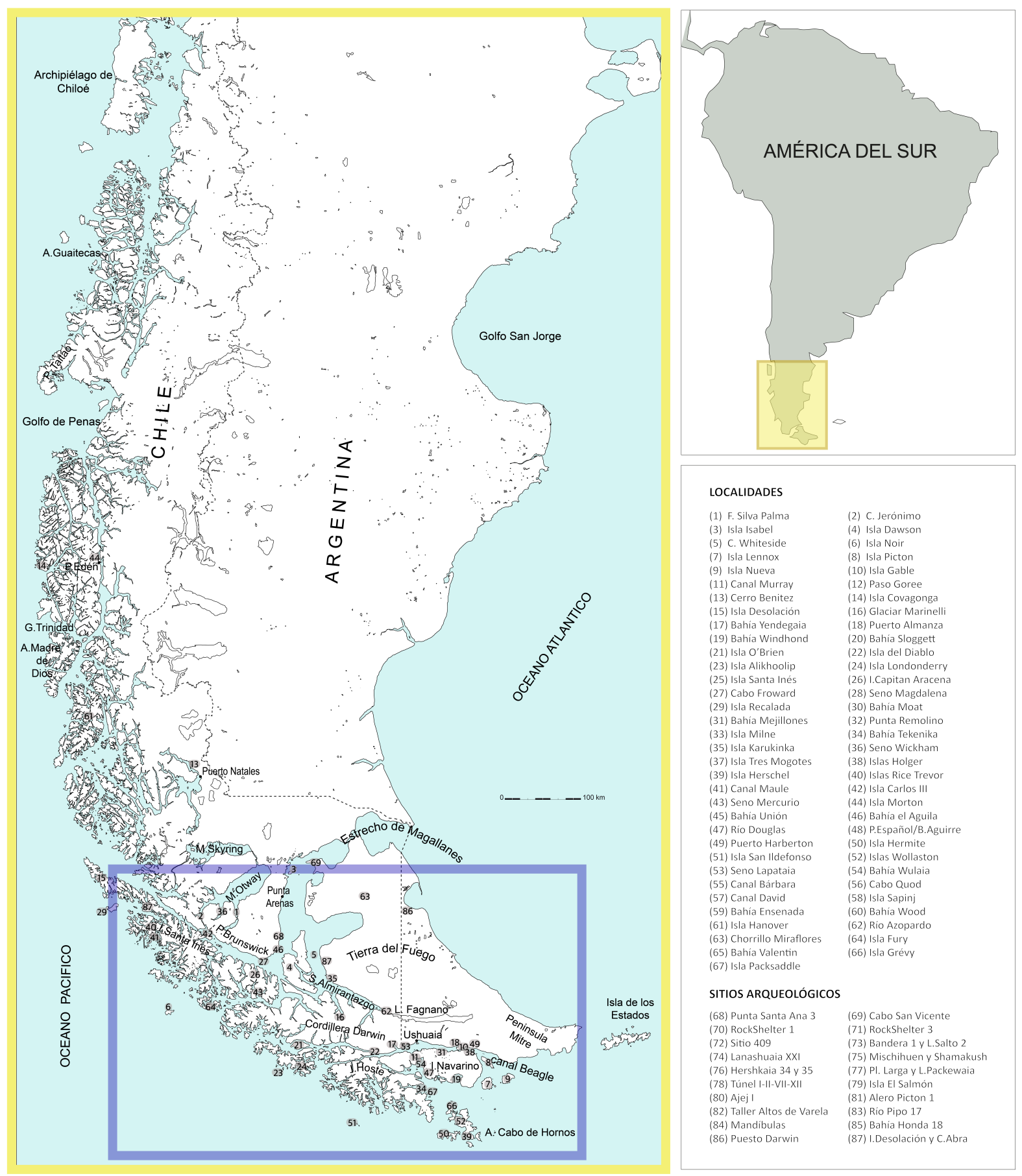

Fig. 1 Localización de Fuego-Patagonia y área de estudio. Recuadro amarillo: Patagonia y Tierra del Fuego. Recuadro azul: archipiélago fueguino. Ver ubicación completa de localidades y sitios arqueológicos en Fig. 2. (Mapa elaborado por V. Sierpe y F. Morello). 
\& Piana, 2015), con una presencia importante de la tundra magallánica (Pisano, 1977). Abundan las observaciones de viajeros acerca de la variable composición de los bosques y de las cambiantes dificultades que ofrecen para la circulación. Skottsberg (2004 [1911], p. 56) menciona el "impenetrable matorral de Nothofagus antarctica /ñire/ ... el agua hasta la rodilla ... árboles caídos, zonas bajas y húmedas y matas espinosas" cerca del lago Fagnano y realiza el contraste con la zona del roble donde "ha desaparecido el bosquecillo denso y verde oscuro con una espesa y empapada alfombra de musgos y helechos; ha desaparecido el canelo" (Skottsberg, 2004 [1911], p. 66). Los espacios abiertos con turberas también ofrecen alternativas para la circulación humana. Asimismo, son cambiantes las condiciones para la navegación y han sido destacadas las dificultades al este de Sloggett (Orquera \& Piana, 1999a, p. 85).

Los ambientes del oeste difieren mucho de los del Beagle central o de la península Mitre. Las turberas son menos extensas, pero topográficamente mucho más complejas. Como resultado, tanto la caracterización zoogeográfica (Venegas \& Sielfeld, 1979) como las características ocupacionales (Aguilera \& Tonko, 2011) difieren significativamente con respecto a los canales interiores, volviéndose la circulación humana más dificultosa.

Debido a razones tectónicas, hay un gradiente este-oeste en el que son cada vez más importantes las condiciones para surgentes (Yesner, 1996), lo que viene asociado con una mayor productividad costera (Matano \& Palma, 2008). Hacia el oeste no hay respaldo de biomasa terrestre significativa en términos de ingesta humana, lo que no es una diferencia menor. La única forma en que se pueden considerar respaldos de biomasa terrestre es por vía de rangos muy amplios de captación de recursos, lo que ya de por sí -en términos de estrategiasconstituiría una diferencia profunda con los casos más orientales y esto es aplicable, igualmente, a las materias primas terrestres exclusivas, como por ejemplo huesos de guanaco (Lama guanicoe) y/o huemul (Hippocamelus bisulcus). Por el otro lado, todo el frente del Pacífico se beneficia con su particular riqueza de recursos oceánicos. La información etnográfica destaca la alta movilidad de los kawésqar que utilizaban las islas occidentales, que podían transgredir los territorios reconocidos (Emperaire, 1963 [1955]; Aguilera \& Tonko, 2011, 2013), capacidad sin duda compartida por los yaganes, muchos de los cuales visitaban regularmente el cabo de Hornos.

La zona oeste de Brecknock se identifica en tiempos históricos con los kawésqar, el canal Beagle y los pasos Murray y Goree con los yaganes y la península Mitre con los haush. En suma, esta gran división ambiental con parámetros tan contrastantes claramente coincide con la división étnica mayoritariamente reconocida desde el siglo XIX, pero se asume que la situación fue distinta para tiempos anteriores, tanto en la circulación humana como en la productividad marina y particularmente la biomasa de mamíferos marinos que fue dramáticamente reducida entre los siglos XVIII y XIX (cfr. Martinic, 1973; Vidal \& Winograd, 1986; Mayorga, 2017). Hacia el oeste se ha considerado la península Brecknock como un límite étnico, pero un análisis detallado del límite occidental de los yaganes en el siglo XIX indica que en tiempos históricos no llegaba hasta allí, pues "había presencia - $y$, al parecer, bastante continuade alacalufes" (Orquera \& Piana 2015, p. 83), sugiriendo como más defendible un límite hacia la isla O'Brien (Orquera et al. 2012, p. 76). En el mismo sentido piensa Chapman, quien considera que en el sector meridional los kawésqar se localizaban entre la península Brecknock e isla del Diablo, con registros de matrimonios mixtos con yaganes (Koppers, 1977 [1924], p. 82; Gusinde, 1991a [1974], pp. 132ss.; Chapman, 2010, pp. 116, 461). Dentro de la escasa información toponímica indígena, la isla Alikhoolip, al sur de la península Brecknock, más precisamente al sur de isla Londonderry, lleva una denominación derivada de la misión del Cabo de Hornos que alude a esta segmentación (Riso Patrón, 1924). Estas zonas "mixtas" en términos étnicos no tienen por qué haber sido fijas y, dada la dificultad para reconocerlas con materiales arqueológicos, justifican nuestro acercamiento, pudiendo verse como un "continuum of groups with intermediate economies" (Suby et al. 2017, p. 176). Estudios moleculares han mostrado diferenciación en los linajes maternales de poblaciones del este y el oeste de los archipiélagos meridionales (Moraga et al. 2010; de la Fuente et al. 2015; Alfonso-Durruty 
et al. 2016). Es posible realizar otras discusiones semejantes para otros sectores de la región bajo análisis. Por ello es que aplicamos el uso de grandes conjuntos culturales, resultantes de la evaluación de nichos ecoculturales; o sea, aludiendo a la distribución de los individuos de una especie o una población dentro de un medio biogeográfico (D'Errico et al. 2017). Esto provee un marco para buscar el rango de condiciones ambientales habitual o potencialmente explotadas/utilizadas por una o más poblaciones (Banks, 2017). En nuestro caso se trata de entidades constantemente cambiantes en múltiples aspectos, que incluyen su número, caracterización e inclusive composición étnica (Borrero et al. 2011). Esta es una forma de avanzar hacia "una visión en mosaico, que contemple cambiantes bloques espacio/tiempo, sin demasiadas asunciones con respecto a las etnias que los utilizaban" (Borrero, 2011, pp. 288289). Asimismo, está en completa sintonía con la fluctuante membresía observada en las distintas unidades sociales de cazadores-recolectores ("bandas"), cuyo funcionamiento se apoya en los sistemas de visitas y otras características organizativas (Williams \& Wobst, 1974; Lee, 1979; Politis, 2007), donde las formaciones multiétnicas no son una anomalía. Utilizamos este enfoque sólo a los fines de plantear una discusión sobre movilidad, extendible a otros ámbitos de la organización social, que abarque todo el sector meridional de la Isla Grande de Tierra del Fuego y el archipiélago fueguino. Así vistas, las variantes postulables oscilan a lo largo de un continuum de adaptaciones marinas a terrestres con varios estados intermedios, cuyo reconocimiento requiere caracterización arqueológica o etnográfica para bloques espacio-tiempo bien especificados. Aquí buscaremos recortar algunas características de los modos de vida manifestados en la región, apuntando a caracterizar la movilidad, la subsistencia y el papel del technium (Arthur, 2009) -formado por todas las tecnologías disponibles pasadas y presentesen la organización social de las poblaciones de los archipiélagos. El technium constituye el eje primordial de la interrelación dinámica entre cultura material (objetos), organización social (procesoscomportamientos) y conocimientos (saberes) de las poblaciones humanas enfocada de distintas maneras por diferentes investigadores. Entonces, los patrones que se observan en diversos registros materiales tienen correlación con aspectos procesuales como la movilidad, estrategias de subsistencia y se vinculan con conocimientos tradicionales de tipo social, tecnológico y ambiental, aunque raramente podamos dilucidar el detalle de estas correlaciones y los factores involucrados (Lemonnier, 1983; Binford, 1991; Pigeot, 1991; Collignon, 1996; Fiore et al. 2008; Arthur, 2009; Whallon, 2011; Whallon \& Lovis, 2016; Borrazzo et al. 2019). Varios de estos patrones alertan acerca de la importancia de algunas diferencias en el espacio o en el tiempo. La demostración de variación registrada mediante estudios morfométricos entre flechas correspondientes a kawésqar y yagán por un lado y a selk'nam por el otro indica una importante diferenciación en la materialización de un instrumento y arma crucial (Charlin et al. 2016), que en el caso de las recuperadas en la zona central del canal Beagle se presentan como muy homogéneas en diseño (Charlin et al. 2014). También debe notarse el contraste entre la relativamente pareja distribución de instrumentos óseos en los sitios conocidos (Christensen, 2016) y la distribución discontinua de cuchillos de concha hechos con Mytilus en la zona central del canal Beagle (Bird, 1932). Asimismo, tomando una perspectiva temporal larga, existe el potencial papel de los fluctuantes costos de manufactura en la discontinuidad decorativa manifestada en armas óseas con un alto riesgo de pérdida (Fiore, 2011). Estos patrones y otros aseguran que tanto a través del espacio como del tiempo son esperables condiciones de habitual relocalización humana y resignificación de materiales en relación con decenas de factores (Binford, 1983a).

También sustentamos nuestro acercamiento en que, aún adentrándonos en el panorama histórico, es posible pensar en la existencia de mayor variación que la normalmente reconocida por la etnografía tradicional, sobre todo teniendo en cuenta los más de 500 años disponibles para dicho período de registros y la desigual cobertura por parte de las distintas disciplinas. Además, desde la perspectiva etnohistórica, se carece de un análisis sistemático de los procesos de transculturación y sus diferencias según zonas eco-geográficas en escalas temporales variadas. Tampoco se ha profundizado en los registros 
etnográficos e históricos de etnias "mixtas", que consideramos aquí como patrones fuera de la norma dicotómica de subsistencias marinas o terrestres y no exclusivamente dependientes del ambiente. La información isotópica muestra que tanto en el canal Beagle como bastante más al este, abarcando la península Mitre, las dietas son predominantemente marinas, a pesar de cierta variación ambiental y presencia de importantes presas terrestres -guanaco- en las islas de Tierra del Fuego y Navarino (Tessone et al. 2011). Los análisis en marcha de la ecología isotópica del canal Beagle ayudarán a valorizar las contribuciones de recursos terrestres a la dieta, incluyendo plantas (Kochi, 2017; Kochi et al. 2017), que es una dimensión poco diferenciada en el registro etnográfico. En otras palabras, la probabilidad de que existan diversos nichos ecoculturales debe ser seriamente considerada. Más allá de las evidencias para resiliencia dietaria medidas por isótopos estables para la arqueología tardía del canal Beagle, extendiéndose hasta tiempos posteriores al contacto (Tafuri et al. 2017), una vez que el análisis se adentra en tiempos previos a Magallanes $y$ abarca todo el abanico ambiental, las posibilidades de variación se hacen enormes. Es dentro de este esquema de configuraciones culturales cambiantes que se plantean las siguientes discusiones sobre movilidad.

\section{EL CASO:}

\section{ARCHIPIÉLAGO FUEGUINO AUSTRAL}

\section{Conectividad y movilidad}

El registro arqueológico del archipiélago fueguino austral es muy disímil, destacando el canal Beagle y el extremo sudeste de la Isla Grande por ser bien conocidos (Legoupil \& Fontugne, 1997; Orquera \& Piana, 1999a; Ocampo \& Rivas, 2000; Zangrando et al. 2011; San Román, 2014; etc.), mientras que la franja occidental, donde se ubican grandes islas como Capitán Aracena, Santa Inés, Desolación, Hoste y otras, ha sido poco estudiada (Emperaire, 1963 [1955]; Gawronski, 1999; San Román \& Morello, 2001; Martinic, 2011) (ver Fig. 2). La caracterización marítima puede ser fácilmente complementada con formas de circulación terrestre en la parte oriental, una posibilidad que se restringe cada vez más hacia el oeste y sur. Se trata de una zona cuya conectividad inmediata debió ser específicamente mediada por las condiciones climáticas y biogeográficas, como la localización del campo de hielo de cordillera Darwin y península Brecknock y otras fluctuaciones de escala tempo-espacial restringidas, como condiciones meteorológicas, corrientes marinas y oscilaciones de marea, entre otras. Incluye las aguas del estrecho de Magallanes, la zona de cabo Froward y seno Magdalena, así como el acceso al mar de Otway mediante el canal Jerónimo o porteos al fondo del fiordo Silva Palma. En general, la conectividad debió ser algo menor que la de zonas más abrigadas hacia el norte y este. Aunque la circulación marítima también presenta cierta diversidad, aumentando hacia el oeste y sudoeste, e incluyendo también las situaciones geográficas más marginales expuestas al océano Pacífico austral en el archipiélago del Cabo de Hornos, la isla Noir y el conjunto de islas cercanas a Recalada. Si bien el foco geográfico regirá la selección de datos, no se omitirán referencias a localidades ubicadas fuera del mismo cuando se las juzgue relevantes, particularmente para introducir aspectos para los que carecemos de información en la zona en discusión.

En este sentido existen algunas generalizaciones inmediatamente defendibles acerca de la geografía cultural de esta región. El modo de vida registrado en tiempos etnográficos en el Beagle central y zonas más al oriente funcionaba a la manera del modelo tradicional de asentamiento de cazadores marinos (Yesner, 1980), presentando distribuciones arqueológicas más o menos lineales interconectadas por agua. David Stuart ha considerado que no sería posible un patrón lineal de explotación de playas sucesivas (Stuart, 1983), pues produciría hacinamiento, además de la posibilidad de llegar a playas ya explotadas, un riesgo que supone que los canoeros querrían evitar. Sin embargo, al menos a lo largo de tramos limitados, eso es lo que parece haber ocurrido. Orquera y Piana (2015, pp. 70-72) hablan de rangos pequeños de desplazamiento de las canoas, lo que está avalado por una variedad de marcadores. Por ejemplo, las materias primas principales son de abastecimiento básicamente local, lo que se une a evidencias de ocupaciones 


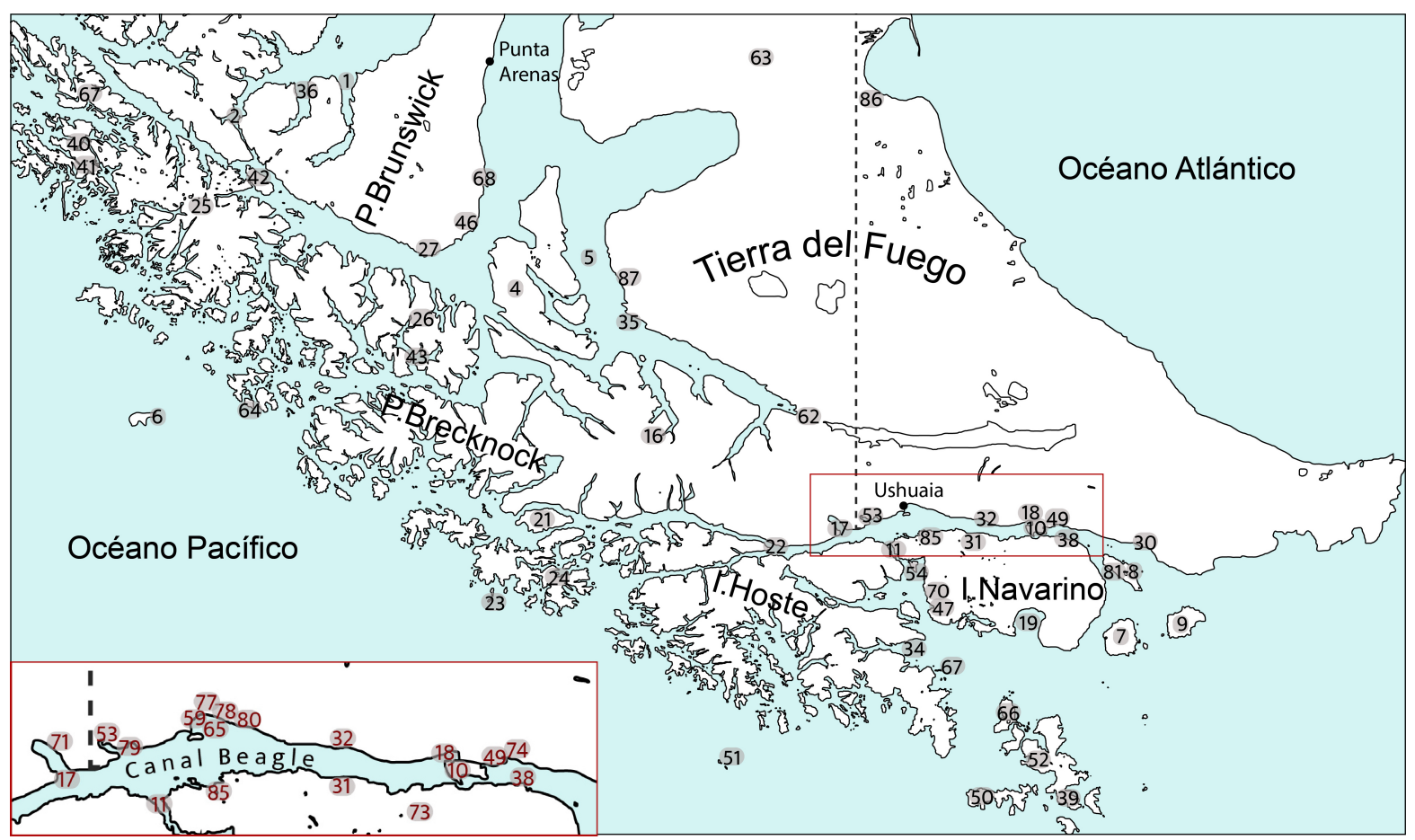

Fig. 2 Distribución de localidades y sitios arqueológicos en el archipiélago fueguino. Recuadro rojo: detalle de canal Beagle. Ver listado completo en Fig. 1.

cortas y redundantes (Breivik et al. 2016). Por otra parte, recientes estudios de Zangrando et al. (2016) sostienen que se puede defender que el pequeño tamaño de Mytilus edulis registrado en el sitio Heshkaia 35, localizado en bahía Moat a unos $200 \mathrm{~m}$ de la línea de costa actual, y datado aproximadamente entre 650 y 500 años atrás, puede marcar una depresión local de los recursos costeros, quizá causada por movimientos periódicos a lo largo de la costa dentro de territorios locales, sin que implique sobreexplotación a largo plazo. Esto parece, dentro de un esquema de "territorial packing", a la vez avalar la observación de Stuart e indicar que no es un problema muy grave.

Por supuesto, esto no inhibe la existencia de cruces del canal, el acceso a islas o el uso de porteos terrestres como complemento de estrategias de conectividad marina. Un primer elemento de contraste es entre movilidad acuática lineal y una presunta movilidad o desplazamiento basado sobre cruces regulares de canales o, en otras palabras, ¿cuál es la importancia del cruce del canal Beagle $\mathrm{u}$ otros cuerpos de agua? La respuesta indica la escala de la conectividad en la región. Durante la estadía de Koppers y Gusinde entre los yámanas hacia 1921, aquéllos tuvieron necesidad de cruzar de Mejillones a Punta Remolino, lo que hicieron con los yámanas, aunque ya usaban una vela, "el tiempo reinante durante algunos días nos impidió emprender el viaje ... los temporales eran tan fuertes y permanentes que nadie, ni los indígenas ... podíamos pensar seriamente en una travesía" (Koppers, 1977 [1924], p. 120). Asimismo, Rosa Yagán cuenta que "a veces el mal tiempo no nos deja cruzar el canal Murray, por eso casi siempre pasamos a la isla Milne ... y de ahí cruzamos a Tekenika" (Stambuk, 2011 [1986], p. 124). Esto marca algunas de las limitaciones -o precisa la escalaque ofrecía la conectividad regional y lleva a pensar los desplazamientos más regulares como lineales. Presenta al canal Beagle como una barrera aun para una sociedad canoera, cuyo tempo está dictado por el clima. Entonces, en el Beagle parece más defendible una movilidad lineal (Zangrando et al. 2016). Para otros canales menores hay referencias de cruces más regulares relacionados con tareas de subsistencia como la caza de guanacos. Es claro que conectividad no implica regularidad en la inter- 
acción, sino sólo la posibilidad de la comunicación y es lo que, en términos metapoblacionales, lleva al "efecto rescate" (Simberloff, 1988). Su importancia para el mantenimiento de la viabilidad de una población es enorme (Grove, 2018).

Sin duda, la principal información etnográfica y arqueológica sobre el asentamiento canoero en los sectores sudorientales de Tierra del Fuego refiere a la costa de islas grandes como Navarino o Grande (Lefèvre, 1993-1994; Legoupil, 19931994; Piana \& Canale, 1993-1994; Legoupil \& Fontugne, 1997; Aspillaga et al. 1999; Orquera \& Piana, 1999a; Piana et al. 2000, 2006, 2008; Zangrando, 2009a; Tivoli, 2010; Álvarez et al. 2013) (Fig. 3), pero también existe información sobre uso de otro tipo de islas. Es menos conocido el uso de espacios más limitados, como los ofrecidos por islas pequeñas como Karukinka, Wickham o Tres Mogotes al norte de Brecknock, o islas como Lennox y Picton en la vertiente atlántica o Herschel, al sur del archipiélago del Cabo de Hornos (Ortiz Troncoso, 1972; Legoupil, 19931994; Aspillaga \& Ocampo, 1996; Legoupil \& Fontugne, 1997; Aspillaga et al. 1999; Muñoz et al. 2016). Al noroeste de la península Brecknock las referencias son más escasas, incluyendo las islas del grupo Rice Trevor en el canal Maule (San Román \& Morello, 2001) u observaciones en las islas Desolación, Santa Inés, Carlos III y Capitán Aracena (San Román \& Morello, 2001; Martinic, 2011; Díaz Lomovasky, 2018). La evaluación de las ocupaciones al sudoccidente de península Brecknock también abarca casos de islas del borde del Pacífico, las que en tiempos históricos configuraron el territorio Tawókser de los kawésqar, asociadas con circulación muy dependiente de las condiciones climáticas (Aguilera \& Tonko, 2011). Esto es así independientemente del grupo étnico considerado, lo que llevó a Skottsberg a decir que las visitas de canoeros al extremo oeste de los archipiélagos para cazar lobos eran improbables y peligrosas (Skottsberg, 1913). Por otra parte, existe información de finales del siglo XIX e inicios del siglo XX que refiere a movilidad indígena de larga distancia, involucrando tanto chonos como chilotes-kawésqar y otras poblaciones híbridas que acostumbraban tener estrategias de alta movilidad por el archipiélago patagónico, en algunos casos incluyendo largos recorridos terrestres que llegaban

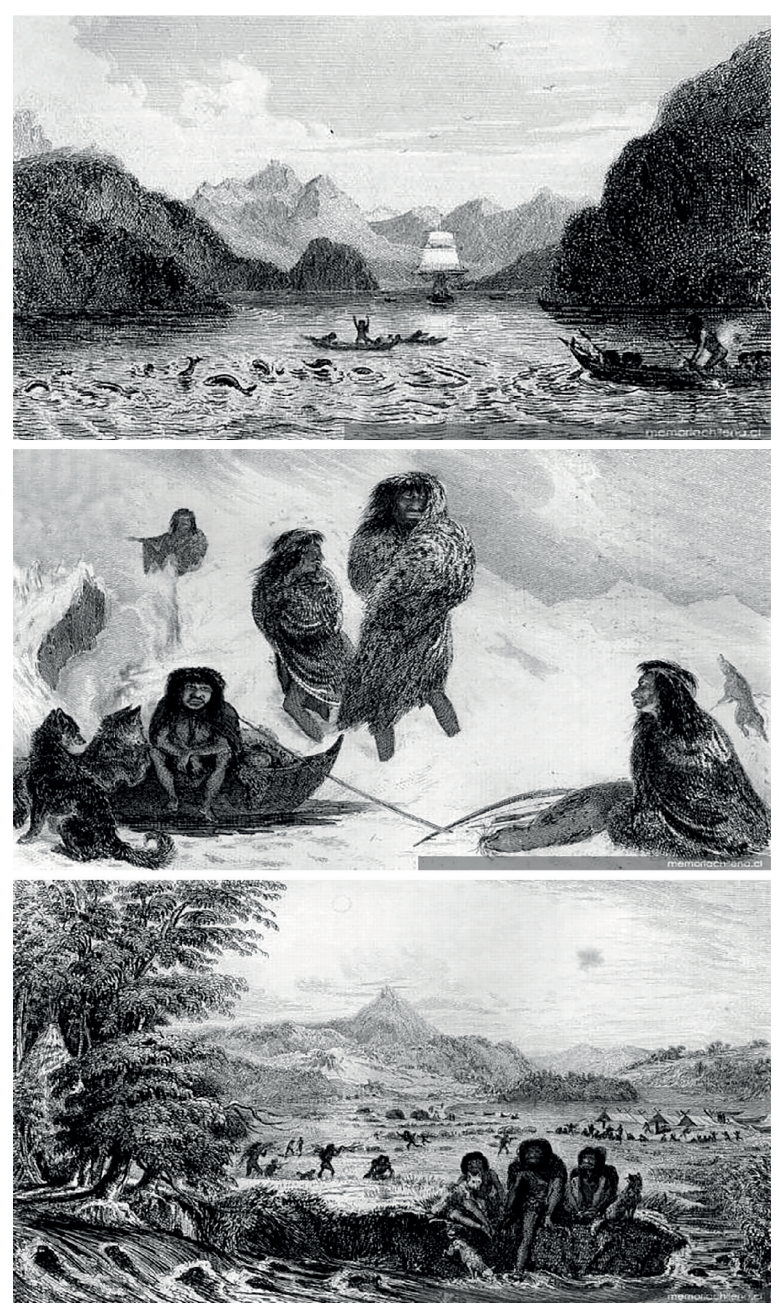

Fig. 3 Dibujos del archipiélago fueguino de Conrad Martens (1933), en Fitz-Roy (1839). Superior: angostura Murray (páginas 326-327); Centro: "Fuegians going to trade in zapallos with the patagonians" (páginas 170-171); Inferior: Wulaia, lámina entre las páginas 208-209. Modificado http://www.memoriachilena.gob.cl/.

a territorio yagán (Bridges, 1952, p. 131; Gusinde, 1991a [1974], pp. 130-134). Esto debió operar como efecto rescate en tiempos de poblaciones diezmadas tanto por enfermedad como presiones sociales y ecológicas. Estos movimientos tenían una orientación preferente norte-sur y conectaban zonas muy diferentes.

Inevitablemente, las limitadas distribuciones de sitios al oeste configuran un patrón diferente al centro de canal Beagle y canal Murray, con estrategias de circulación más complejas -no lineales- que muchas veces incluyen porteos, o sea, 
sectores terrestres preparados y/o seleccionados para circular a pie transportando las canoas. La distribución pautada por islas y canales de los canoeros del oeste y sur de Tierra del Fuego, a priori mucho más asimilable a ocupaciones no alineadas, generando distribuciones con poca continuidad geográfica, debió posibilitar adaptaciones probablemente diferentes a las observables en el canal Beagle central. En los archipiélagos los canoeros occidentales disponían de conectividad metapoblacional, por lo que en la línea de Cane (1990) pueden fácilmente ser considerados casos de pequeñas agrupaciones con infrecuentes contactos y altos niveles de autonomía, que pudieron pasar períodos en relativo aislamiento. Seguramente esta situación no implica condiciones de homogeneidad $y$ estancamiento como las que vio Emperaire en momentos muy tardíos (1963 [1955], p. 112), pues la conectividad relativamente limitada se asocia normalmente con tendencias a la innovación (Derex \& Boyd, 2016). Sin embargo, la continuidad de estos grupos vía efecto rescate pudo ser variablemente efectiva, muy dependiente del clima, lo que invita a pensar que las condiciones de sobrevivencia pudieron ser más dificultosas. Esto no significaría que estuvieron al borde del colapso, sino que la organización de los movimientos y encuentros considerando un conjunto amplio de variables debió ser muy estricta.

La importancia de las tormentas en la región es muy conocida y su combinación con zonas de corrientes entre canales y fiordos se manifiesta de diversas maneras, tales como mar de leva, williwaus, granizadas o vientos encajonados (Tuhkanen et al. 1989-1990; Pallo, 2011). Aspectos del modelo de tormentas, que pueden dificultar la elección de los lugares de arribo de las canoas (Stuart, 1983), pueden aplicar mejor en estos sectores occidentales, muchas veces dominados por amplios acantilados. Piana $y$ Orquera (1998, p. 421) sostienen que el modo de traslado acuático era superior al terrestre a pesar de las tormentas. Sin duda era superior, con rangos diarios de acción potencialmente más grandes que los terrestres (aunque ver Breivik et al. 2016, p. 90), lo que no quita que tuviera sus limitaciones, mínimamente incluyendo la construcción, mantenimiento y reemplazo del utillaje marítimo, todas tareas costosas. En otras palabras, el éxito de estas adaptaciones occidentales pudo ser comparativamente más costoso que en el oriente.

\section{Conocimiento y movilidad}

Muchas veces la evaluación de la subsistencia de cazadores-recolectores ha girado alrededor del concepto de predictibilidad. Sin embargo, Binford (1983b) ha destacado que la predictibilidad no es una característica de un ambiente, sino una propiedad táctica relacionada con el grado de conocimiento del ambiente. Dado un buen conocimiento, todo ambiente es predecible. De manera que el foco se desplaza hacia el grado de conocimiento trasuntado por los registros etnográficos o arqueológicos. Para el período en cuestión, todo parece indicar que nos referimos a poblaciones que disponían de buen conocimiento de los recursos locales, que enfrentaban variadas condiciones de riesgo, pero que en su interacción con los recursos debían mantenerse atentas a situaciones favorables o desfavorables no planificadas con precisión, en otras palabras, a lo que se denomina incertidumbre (Binford, 1991, p. 35). Las hambrunas han de contarse entre estas situaciones (ver abajo).

\section{Moluscos y subsistencia}

La posición dominante acerca de la subsistencia ha sido que se basaba en "sea food and seal" (Cooper, 1917, p. 187) y se mostró que los moluscos no eran el recurso dominante (ver discusión en Orquera \& Piana, 2015, pp. 161ss.). Aún menos lo eran otros invertebrados como crustáceos y equinodermos (Santiago \& Vázquez, 2009). Por otra parte, los restos de aves raramente son dominantes, pero son importantes (Piana \& Canale, 1993-1994; Piana et al. 2008; Tivoli, 2010). Ese papel menos central de los moluscos es muy defendible, aunque siempre debe recordarse que es difícil sostener que el consumo de moluscos en el pasado puede ser medido por el cálculo de lo que se ha recuperado en concheros (ver Orquera \& Piana, 1996, pp. 237ss.). Los pocos casos de observaciones etnoarqueológicas sobre formación de concheros muestran que muchas veces una parte 
de lo que se consume se descarta directamente en el campo (Meehan, 1982, p. 117). Un caso especial de bienes exóticos es creado por el transporte diferencial de moluscos. Al respecto corresponde repetir el clásico testimonio de Byron, quien en 1742 registró en el archipiélago Guayaneco que los canoeros comían y guardaban las conchas en el fondo de la canoa, las que llevaban a la playa y colocaban sobre la línea de alta marea (Byron, 1996 [1768], p. 89). Esto también aplica en el canal Beagle en los casos en que los yámanas comían a bordo de sus canoas, "los residuos no debían ser arrojados al agua, sino quemados en el fuego que ardía en el medio de la canoa ... o guardados hasta llegar a tierra" (Orquera \& Piana, 2015, p. 253; ver también Gusinde, 1986a [1937], pp. 1280, 1283; Stambuk, 2011 [1986], p. 83). Finalmente, muchos moluscos recolectados y depositados en concheros pudieron no haber sido consumidos, como se ha sugerido para algunos de tamaño muy pequeño quizá resultantes de la recolección de "racimos" de moluscos (Zangrando et al. 2014). En otras palabras, los cálculos acerca de proporciones de alimentos a partir de hallazgos en sitios son poco confiables. No sólo enfatizan los huesos y restos más resistentes, sino que -aun suponiendo que los estudios den cuenta de la conducta de dividir las presas (Enloe, 2010)- ignoran aquello que no se depositó en el sitio. Independiente de su importancia en la dieta los moluscos constituyen el recurso más ubicuo de los canales fueguinos, es el recurso de "salvataje" diario que implica menor riesgo y altísimo potencial de éxito por lo que adecuadamente ha sido considerado una especie de "válvula de seguridad" para la subsistencia (Orquera \& Piana, 2009, p. 70). Los procesos de formación, con la consideración de mezclas de restos no relacionados con el depósito arqueológico, también deben ser muy tenidos en cuenta (Alunni et al. 2017). La discusión requiere, en todos los casos, un marco regional. Sin duda, la herramienta metodológica adecuada para avanzar en este tema son los isótopos estables en muestras humanas (Yesner et al. 2003; Tessone et al. 2011; Kochi, 2017; Tafuri et al. 2017). Todos estos análisis proceden por el momento con una mínima base cronológica, la que sin duda se irá refinando y que es particularmente necesaria para evaluar la situación pre y post contacto.

\section{Cuchillos de concha}

La utilización de moluscos para la confección de herramientas utilitarias se restringe a los cuchillos de concha y definen la "shell-knife culture" que Junius Bird identifica en sitios de los canales Beagle y Murray. Estos Mytilus gigantes, sin claridad sobre la especie particular, tienen una disponibilidad limitada (Bird, 1938, p. 59) y existe sólo un dato de lugar de aprovisionamiento en el archipiélago fueguino, en la isla Packsaddle: "Large Knife mussel: The only bed of these known to the Yaghans is down by Packsaddle Is. Milisich, [José Milicic, marido de Rosa Yagán] while down otter hunting, gathered only a dozen or so shells. He prized these so much that he would only give Ken one for me. The mussel had been cut out of the shell because roasting it free might reduce the hardness" (Bird, 1932, p. 183). Son escasos los cuchillos de concha arqueológicos conocidos (Bird, 1932; Figuerero Torres \& Mengoni Goñalons, 1986; Morello et al. 2018).

\section{Utilización y consumo de plantas}

Existe muy poca evidencia arqueológica para evaluar el consumo de plantas en los canales sureños, pero es abundante la información etnográfica al respecto (Piqué, 1999; Berihuete Azorín, 2014). En particular, se ha destacado la importancia de los hongos (Bridges, 2001). Desde ya hay un lugar importante para los recursos vegetales con fines no alimenticios, como la fabricación de arcos con madera de Nothofagus betuloides (Ratto, 2003) o de astiles de flecha con Berberis (Ratto \& Marconetto, 2012). Se ha observado una "recurrencia en la mención del recupero del astil durante más de 100 años de observaciones" (Ratto \& Marconetto, 2012, p. 138), lo que es una información en términos de los costos de estas adaptaciones que puede incluirse a la par de la discusión de los cambios en patrones decorativos de armas óseas (Fiore, 2011). Ampliando el estudio de colecciones etnográficas hubo además una confirmación del uso de Nothofagus sp. para arcos y de la preferencia por Berberis para astiles, con el agregado de Ribes magellanicum (Caruso Fermé et al. 2011). Recientemente Maerky inició el estudio de mangos de madera kawésqar y 
yagán considerando las propiedades de diferentes maderas locales (Maerky, 2018). Todo esto apunta a esperar condiciones de abastecimiento local, que no requerían grandes desplazamientos. Orquera et al. (2012, p. 51) informan sobre una experiencia comparativa acerca de la resistencia de corteza y madera de Nothofagus y madera de Austrocedrus chilensis, que ayuda a comprender la elección de corteza de Nothofagus para construir canoas, lo que en algunos casos implica una forma de abastecimiento no estrictamente local para un ítem crucial en la adaptación a los canales.

Con respecto a los fuegos, los ambientes del sur de Tierra del Fuego presentan abundancia de leña, por lo que hubo uso intenso de maderas costeras locales (Caruso Fermé \& Zangrando, 2018). Además, son abundantes los registros etnohistóricos y etnográficos sobre las técnicas implementadas para mantener el fuego constantemente encendido en chozas y/o canoas (e.g. Gusinde, 1986b [1937]; Orquera \& Piana, 1999b; Duplessis, 2003 [1699-1701], entre otros). El análisis de fitolitos en el sitio Túnel VII, asignado al período 1776-1898 AD, identificó madera quizá usada como combustible (Zurro et al. 2009, p. 189), mientras que la antracología mostró el dominio de Nothofagus betuloides y Maytenus magellanica (Piqué, 1999; Zurro et al. 2009, p. 186). Desde la antracología se ha interpretado que en algunos casos es posible "una adaptación [...] para la recogida de leña, en respuesta a las primeras transformaciones del mundo forestal" (Solari, 1993-1994, p. 147), concretamente desde la elección de Nothofagus betuloides a la de Drimys winteri. Esto, junto a las múltiples evidencias de fuegos (por ejemplo, Weddell, 2006 [1825], p. 169), es un importante elemento para discutir los procesos de construcción de nicho por parte de poblaciones con alta movilidad.

\section{Recursos y planeamiento}

Si bien resulta necesario profundizar en los componentes vegetales de la dieta-cuyas dificultades de preservación han sido discutidas en detalle por Berihuete Azorin (2009)-, debemos reconocer que, en lo alimenticio, el mundo de los canales -sin llegar a los extremos de algunas sociedades periárticasbásicamente es un mundo de carne y grasa. Parece improbable que los vegetales hayan sido la razón de movimientos específicos de gente. Tampoco existen problemas importantes de acceso a los recursos dentro del ciclo anual del sector oriental de Tierra del Fuego, aunque sí podría haberlos en el sector occidental debido a la falta de biomasa animal terrestre. De todas maneras, el tiempo de aparición de los recursos puede presentar-debido al clima, ciclos ecológicos, variaciones causadas por intermareales exagerados, períodos de tormentas, inviernos muy crudos- mínimas diferencias año a año que -en sociedades sin almacenamiento a gran escala- crean pequeñas, pero quizá significativas incongruencias.

También debe considerarse un aspecto estacional, como una mayor dificultad para encontrar recursos en invierno (ver otra opinión en Orquera et al. 2012, p. 101). Varias incongruencias coexisten en los canales, ante todo la espacial de colectar moluscos en la costa y cazar lobos en aguas abiertas (Stuart, 1983), que de acuerdo con la etnografía ha tenido una resolución a través de la división sexual del trabajo. Esta resolución además ha de tener una dimensión temporal, en otras palabras, horarios de caza y horarios de recolección en función de las mareas y las condiciones meteorológicas. También deben considerarse algunas incongruencias temporales, como las relacionadas con las escasas plantas utilizadas y sus limitadas ventanas de uso, o la relacionada con la distribución costa-interior de guanacos. El manejo de estas incongruencias requiere un planeamiento que no ha sido totalmente puesto en evidencia por la etnografía o la arqueología, muchas veces oculto bajo una imagen de cazadores-recolectores que vivían al día. La existencia de almacenamiento de alimentos, por limitado que sea, ya alerta acerca de estas alteraciones a la disponibilidad continuada de recursos.

\section{Hambrunas, abundancia y circulación}

Los casos de hambrunas son bien conocidos durante tiempos históricos en los canales (Darwin, 1906 [1839], p. 203; Cooper, 1917, p. 187; Chapman, 2010, pp. 367, 464-465; Aguilera \& Tonko, 2011, p. 58). De hecho, son muchas las referencias a hambrunas, llegando a casos de "comerse el cuero de sus tiras" (Hyades \& Deniker, 
2007 [1891], p. 87) o al comentario de Gusinde sobre la pesca con corrales como paliativo ante situaciones de hambruna o la imposibilidad de desplazarse, que en el fondo es lo mismo (Vázquez \& Zangrando, 2017, p. 105).

Para el caso de los kawésqar, Gusinde (1991a [1974], pp. 129, 330) dice que no pasaban hambre. Sin embargo, Aguilera y Tonko (2013, pp. 265ss., 305) sí lo mencionan entre los kawésqar del sur. Yesner (1980, p. 729) enfatizó la importancia de lo que Birdsell llamó "unearned resources", básicamente recursos migratorios o que vienen de otros lugares rendidores, aunque como este autor destaca a veces pueden ser costosos. Para Yesner (1996) un ejemplo fueguino son las ballenas varadas. Muchas veces estos casos son un buen ejemplo de abundancia de un recurso y han sido asociados con ingestas excesivas (Chapman, 2010, p. 466), la contrapartida de las hambrunas. Estos momentos de abundancia no se restringían a casos de ballenas. Junius Bird destacó una situación en que "found the shore as far as the next point west just covered with gulls, as thick as they could be. The seals were feeding on small fish like sardines and the gulls were picking up what the seals missed. Shot 6 young gulls and the whole outfit of gulls and seals went off. On returning to camp found them all there and schools of the fish swimming just in front of the tent. Peeled off the pants and went after them with the butterfly net, filling it to the brim at each scoop. Caught a small barrelful in no time. One could have caught them with a bucket just as well. The schools keep swimming in a circle 30 or 40 feet across and when one wades rapidly in amongst them they pile upon each other and form almost a solid mass. In water about 4 feet deep they are so thick they get under your feet when you walk. At the beach just to the east the seals came inside the kelp to get at the fish and about a dozen seals were swimming back and forth there for several hours. Walked almost to the edge of the beach abreast of them without frightening them. An Eskimo could easily have harpooned them from the shore, they were so close" (Bird, 1932, p. 164).

También abundan las referencias a depósitos de carne y grasa de ballena derivados de períodos de abundancia. Una observación realizada bastante más al norte es muy informativa con respecto a las soluciones ante el hambre. En ocasión de una hambruna causada por el mal tiempo un grupo de personas que Fitz Roy denomina Chono (en realidad kawésqar, como señalan Quiroz et al. 2016) sale a buscar alimento y retorna cargado con grandes trozos de grasa podrida de ballena (Darwin, 1906 [1839], p. 203; Álvarez Saldivia, 2016, p. 109; Fitz Roy, 2016 [1839], p. 180), que estaban en un depósito. Es precisamente la existencia de estos depósitos lo que indica un grado de inseguridad en el abastecimiento de alimento (Martial, 2005 [1888], p. 224). Para Úrsula Calderón los yaganes de Cabo de Hornos pasaban el invierno donde se localizaba alguna ballena (Chapman, 2010, p. 52), hallazgo que según Gusinde -entre otros- se anunciaba con humo (Gusinde, 1991a [1974], p. 185), práctica que ha sido puesta en duda por Tonko (2019). Esto podría significar que no había tantos recursos fáciles de obtener en invierno. Es reconocido, además, que el varamiento era una circunstancia adecuada para que la gente se concentrara en ceremonias (Chapman, 2010, p. 56).

Las referencias al hambre incluyen, lógicamente, lugares alejados como las islas Wollaston, donde Burleigh menciona hasta unos 15 días sin poder buscar comida debido al mal tiempo (Orquera \& Piana, 2015, p. 184). Cuando los problemas ocurrían en ese u otros lugares alejados las situaciones no eran fáciles de resolver; cualesquiera fuera el ritmo del uso de esas islas, alejarse implicaba travesías a mar abierto y coincidían, por supuesto, los períodos de escaso alimento con las tormentas. Estos casos en esas u otras islas alejadas sólo pueden verse como críticos y significativos para la viabilidad de subpoblaciones, que ven limitada su capacidad de circulación.

Se habla de una gran densidad humana en el canal Beagle, unas 30-40 veces mayor que en Patagonia o Pampa (Orquera \& Piana, 2015, p. 70), aunque también se ha sugerido que la imagen de alta densidad es el resultado tanto de la abundancia de sitios arqueológicos registrados como de la atracción ejercida por las primeras naves europeas (Borrero, 2002). De todas maneras, por alta que fuera la densidad, aparentemente no implicaba saturación del espacio. La literatura está llena de ejemplos de playas y otros sectores vacíos, observaciones sin duda válidas sólo en el corto plazo, pero que inevitablemente implican ausencia de saturación del espacio. Asimismo, el misionero Gardiner y su gente pudieron hallar un 
lugar desolado -Puerto Español, localizado más al este- para sus últimos días, donde murieron de hambre. Este lugar fue elegido porque era raramente visitado por indígenas, fueran canoeros o terrestres (Bridges, 1951, p. 39; Chapman, 2010, p. 294). En general, el panorama es de mayor discontinuidad ocupacional al sudoeste de Brecknock, probablemente asociado con un conocido cambio en la distribución de la productividad, quizá desde fines del siglo XVIII (Vidal \& Winograd, 1986; Mayorga, 2017). Es aceptable que pudieron ocurrir cambios previos, relacionados entre otras cosas, con los ritmos ecológico-climáticos y las actividades humanas.

La imagen de alta demografía quizá llevó a relativizar las historias de hambrunas entre los yámanas. Han sido vistas como situaciones transitorias, creadas por tormentas, canoas perdidas u observaciones hechas en la Misión Anglicana, sosteniéndose que "el año no incluía temporadas de escasez crítica” (Orquera \& Piana, 2015, p. 71), lo que es totalmente cierto. Sin embargo, como aclaran Orquera y Piana, "es indudable que los Yámanas estaban sometidos a períodos de hambre" (2015, p. 188). Una referencia recogida por Junius Bird es pertinente, "Clemente says mussels are not eaten in December and first part of January, whey they are very thin and watery. They are not poisonous or disagreeable but just 'cook to nothing, all water'. The Tapor-mauchos- are the same in September. One winter Clemente says be had nothing but mussels and ate a five gallon gasoline tin full of them every day" (Bird, 1932, p. 149).

En la discusión acerca de las hambrunas se ha mencionado que las fotos de yaganes tomadas en tiempos en que ya era defendible una condición de hambre por escaso acceso a carne de lobo -considerada a partir de ca. 1880 (Orquera, 2002), aunque bien podría ser desde finales del siglo XVIII (Chapman, 2010, p. 113; Mayorga, 2017, 2018)prueban que "no muestran señales de desnutrición y, por el contrario, varios individuos obesos" (Orquera \& Piana, 2015, p. 71). Pero las fotos no son un buen marcador, pues se ha destacado que los yámanas cambiaban de figura en horas (Hyades \& Deniker, 2007 [1891], p. 124). Una situación similar ha sido apuntada para el área kawésqar, aunque más al norte. Tras comer mucha carne y grasa de ballena "la familia vuelve ... completamente transformada: la asimilación debe ser particularmente rápida y sus efectos duraderos, pues todos se mantienen largo tiempo en estado floreciente. Los niños ... se ponen irreconocibles por la capa de grasa que acumulan bajo la piel" (Emperaire, 1963 [1955], p. 122). Más importante aún, atravesar períodos de hambre no implica necesariamente malnutrición. Dichos períodos pueden ser parte de los elementos percibidos del hábitat, constituyendo un riesgo, un elemento de la geografía cultural.

Puede ser perfectamente defendible que las hambrunas sólo ocurrieron en escasas situaciones, en algunos casos tal vez causadas por la instalación de la misión. Efectivamente, durante el período de la Misión Anglicana abundaron los momentos de hambre, en especial durante los inviernos de 1874 y 1875 (Chapman, 2010, pp. 464-469; Orquera \& Piana, 2015, p. 70). Hay registro de hambrunas aun durante el invierno suave de 1889 (Chapman, 2010, p. 554). Al respecto escribió Thomas Bridges (2001, p. 37), "creo que han visto muchos días sin comida". Estos problemas eran mínimamente solucionados con nabos, sopas, té y, en algunos casos más efectivos, con ballenas varadas, incluyendo un caso a unos 74 km de Ushuaia (Chapman, 2010, pp. 464-465). Cristina Calderón recordó períodos de hambre en los años 1930 (Zárraga, 2016, pp. 37, 47). De todas maneras, aunque sean los únicos ejemplos -no lo son- siguen siendo hambrunas y continúan produciendo efectos. Desde ya que importan las causas al evaluar la trayectoria de las sociedades, pero los efectos son más importantes. Finalmente, Thomas Bridges ha consignado muertes por inanición (T. Bridges, en Orquera \& Piana, 2015, p. 112). También Hyades y Deniker consignan un caso en que los nativos pierden la canoa en una isla y mueren de hambre y frío (2007 [1891], p. 97). Debe enfatizarse que en yagán había palabras para la muerte por inanición, como tamashagamaala (Orquera \& Piana, 2015, p. 183). El tema de las hambrunas no implica que las sociedades en los canales estaban amenazadas de extinción, pues estas condiciones sólo aplicaban a subpoblaciones, o inclusive a una escala menor, pero sí debe aceptarse que constituían parte de los ritmos vitales.

Una hambruna transitoria puede ser un problema para la continuidad de un grupo de gente. Una de las causas listadas, las tormentas, 
podía presentarse con excesiva regularidad, como lo indican las referencias etnográficas y los relatos de viajeros. El comentario "demasiado a menudo una familia se ve impedida de seguir su viaje por las inclemencias del tiempo" (Gusinde, 1986a [1937], p. 1283) es muy creíble. Se agrega el realismo de los inviernos duros. Bridges menciona casos de "frozen channels ... impossible to use canoes for catching fish .... Cut off all supplies of mussels and limpets; when guanacos in their hundreds had died of starvation" (Bridges, 1951, pp. 354-355; Fernández et al. 2018), así como debe recordarse su referencia a "a succession of hard, prolonged winters" (Bridges, 1951, p. 355). Asimismo, "cuando los malos tiempos tan frecuentes en estas regiones, duran mucho, la pesca se hace imposible y los escasos recursos guardados se agotan pronto" (Martial, 2005 [1888], p. 224), creando situaciones potenciales de retracción o abandono de lugares. El panorama temporal del tema de las hambrunas necesariamente incluye una evaluación del status de los ambientes siempre expuestos a inestabilidad o a presiones ecológicas y sociales periódicas (Bishop, 1978; Minc, 1986; Speth, 2010). El panorama temporal constituye un componente fundamental de la geografía cultural. Estas situaciones se agravan en tiempos de tormentas fuertes y/o duraderas o eventos climáticos como los correspondientes a la Pequeña Edad del Hielo (PEH), que pudo llevar a las poblaciones a innovar, adaptar tácticas y estrategias previas o acudir a tecnologías latentes. Se ha asumido que el período reciente no ha sido de grandes cambios climáticos (Briz Godino et al. 2011). Sin embargo, esto es relativo a los momentos temporales que se comparan y ha sido revisado por Koch (2015), entre otros trabajos que han mostrado que los glaciares de la cordillera Darwin experimentaron avances durante ese período. Asimismo, Ponce et al. (2011 muestran que el registro polínico de bosque de los últimos 500 años declina notablemente en Isla de los Estados, lo que interpretan como respuesta al frío y vientos de la $\mathrm{PEH}$, período para el que se reconoce un importante descenso de las temperaturas (Fernández et al. 2018). Todo esto parece una lectura más realista de los resultados de Obelic et al. (1998). Bastante más al oeste se ha caracterizado al período final del Holoceno tardío como de grandes fríos durante la PEH (Caniupán et al. 2014). Igualmente, el establecimiento de las oscilaciones modernas de El Niño (ENSO), a partir de ca. 3.000-4.500 años AP, permite considerar que estas fluctuaciones también pudieron ser importantes y potenciar otras, con repercusiones en distintas escalas temporales (estacional, anual, decadal, secular). Estas oscilaciones representan la más extensa perturbación climática de escala interanual del globo y presentan variación espacial, por ejemplo, entre costa Pacífica y mares interiores (cfr. Carre et al. 2014). Estas situaciones de posibles cambios climáticos en plazos cortos, que sin duda debieron afectar los patrones de movilidad, habilitan la futura exploración de bloques espaciotiempo mejor definidos en función de cambiantes nichos ecoculturales y llevan a la consideración del uso de estrategias de control de riesgos en zonas de concentración de recursos (Ozán \& Pallo, 2019).

En síntesis, no hay duda de que los canales presentaban riqueza alimenticia, pero no bastaba que hubiera mucho alimento disponible, era preciso acceder al mismo. Los náufragos suelen navegar o flotar sobre una enorme riqueza de alimentos, pero su problema es que no logran acceder fácilmente a ellos. En general, no pueden cazar las ballenas o siquiera los delfines. Recordemos, por ejemplo, que los delfines no han sido importantes como presas de caza en el canal Beagle (Orquera \& Piana, 2015, p. 133), sosteniéndose que, "canoes and boats are too slow to catch dolphins" (Piana, 2005, p. 132), una observación que es al menos concordante con los inútiles intentos de capturar marsopas por parte de balleneros náufragos (Chase, 2000, pp. 59, 63). En tiempos etnográficos, tanto ballenas como delfines han sido obtenidos en Tierra del Fuego básicamente por varamientos. En contraste, aunque con el foco en otro período, San Román (2016, pp. 201-202) destaca que las localizaciones de sitios con restos de delfín o de marsopa se orientan a sotavento de los vientos predominantes, "factor que inhibiría el varamiento", los que, por otra parte, consignados por Gibbons et al. (2000), no se registran en las listas de varamientos regionales. Esta corta discusión nos lleva a sugerir que la principal razón para que se presentaran hambrunas no derivaba de una pobreza ambiental, sino que era simplemente una expresión de la geografía cultural de la región. En otras palabras, de la relación establecida entre recursos, seres humanos y conocimientos. 


\section{Intensificación del contacto occidental}

El impacto ecológico de la sobrecaza de mamíferos marinos, circulación de naves extranjeras y asentamiento de colonos, modificando la subsistencia, la movilidad y el technium regional empeoraron o al menos desacoplaron esas condiciones alargando los ciclos ecológicos negativos y entorpeciendo la implementación de estrategias previamente útiles. La disminución en la disponibilidad de recursos críticos, o sea aquellos necesarios para completar el ciclo anual, derivada de estos u otros factores, sean previos o posteriores al contacto, conlleva cambios de estrategias, una de las más reconocidas son los procesos de intensificación (Lupo et al. 2013), los que por cierto han sido identificados en el extremo sur a través de un incremento de los esfuerzos dedicados a la pesca (Zangrando, 2009b).

En cuanto a los contactos entre las etnias locales y loberos o balleneros, las discusiones se han concentrado en los viajeros más reconocidos, pero existe un riquísimo historial de contactos, incluyendo los considerados "encuentros fugaces", cuya importancia no está aún totalmente explorada (Mayorga, 2018) y que regularmente resultaban en acceso a los barcos, obtención de objetos de metal o vidrio y una variedad de interacciones. El tempo de estas interacciones claramente se hizo más intenso a partir de los proyectos misionales (Chapman, 2010). El resultado neto fue un notable desacople entre los modos de vida locales con cierta profundidad temporal y las cambiantes condiciones ecológicas y culturales.

\section{DISCUSIÓN}

Kenneth Ames sugiere que el transporte logístico marítimo se facilita en velocidad, radios y cargas (Ames, 2002), lo que también ha sido evaluado para las costas del canal Beagle (Piana \& Orquera, 1998). En este último caso se ha destacado que el uso de botes no sirvió para incrementar la distancia de los rangos (Breivik et al. 2016, p. 90), aunque eso pudiera ocurrir ocasionalmente. Es llamativo el caso de Julia (Carrupale-Kipa), yagán, que escapó de la misión del río Douglas, ca. 1910, remó en canoa monóxila durante casi dos meses, unos $450 \mathrm{~km}$, junto a su perro, alrededor de la isla Hoste, hasta bahía Mejillones (Chapman, 2010, pp. 588589). Julia eligió un camino indirecto, largo, en lugar de usar el paso Murray. Asimismo, Piana y Orquera (1998, p. 422) citan a Stirling, acerca de un hombre que recorrió $25 \mathrm{~km}$ en una noche; y a Lawrence, acerca de un hombre que recorrió 65 $\mathrm{km}$ en dos días. Con mal tiempo esos recorridos eran más lentos, existiendo un registro de cuatro días para cubrir la distancia mayor a $65 \mathrm{~km}$ entre Harberton y Ushuaia; también se conocen varios casos de travesías a mar abierto hacia las islas Hermite o San Ildefonso (ver Orquera \& Piana, 2015, p. 255), lo que podríamos llamar rangos mínimos requeridos para asegurar conectividad entre islas muy separadas entre sí. La expedición en kayak de Barry Smith de 1978 tardó 12 días desde Puerto Williams a Cabo de Hornos, vía canal Murray, islas Hoste y Wollaston. En su retorno la expedición cruzó desde las islas Wollaston a Navarino y desde allí lo hizo hasta el paso Goree (Smith, 1979). Los cruces a mar abierto fueron los que requirieron mayor planificación, insumiendo un tiempo de espera. Se ha sostenido que el modo de vida cazadorrecolector no podía basarse en planificación (Gamble \& Gaudzinski, 2007, pp. 160-161), pero ante situaciones como las indicadas parece difícil respaldar que no la incluyera (Koppers, 1977 [1924], p. 120; Stambuk, 2011 [1986], p. 124 , entre otras). La geografía cultural de una región implica la existencia de conocimiento acumulado, sea situacional o no, cuyo manejo constituye planificación. Por esa razón es difícil aceptar el concepto de visitas de lugares al azar (Breivik et al. 2016, p. 88) o el de arribos no planeados de canoas (Stuart, 1983) como características sistemáticas de la geografía cultural fueguina. Debe enfatizarse que la planificación del movimiento puede verse como una evaluación de riesgos, con el uso del conocimiento disponible para estimar la posibilidad de retorno al lugar de partida, llegada al lugar de destino previsto en el tiempo requerido y posibilidades alternativas de paradas/movimiento.

En contraste con las observaciones clásicas acerca de alta movilidad en el canal Beagle y alrededores (por ejemplo, Fitz Roy, 2016 [1839], p. 167), Yesner sostuvo que los sitios de Playa 
Larga representaban relativamente poca movilidad, hablando de "longer-term occupation than the three to four days suggested by ethnohistorical accounts" (Yesner, 1990, p. 16). Ese registro también puede verse como resultado de alta redundancia ocupacional, alternativa que admite (Yesner, 2004). En favor de permanencias más largas esta discusión puede invocar movimientos de las chozas por razones de higiene (Hyades \& Deniker, 2007 [1891], p. 91) u otros motivos. Bastante más al este, en bahía Valentín, los estudios de Scheinsohn sobre la variedad de diseños de instrumentos óseos hacen atractiva la exploración de que se tratara de un lugar de agregación (Scheinsohn, 2011).

Se ha sugerido -sobre la base de análisis zooarqueológicos- que para los tiempos posteriores a 1.500 años AP (Zangrando, 2009a) hubo una reorientación en el uso del espacio, incluyendo ampliaciones de rangos de movilidad marítima(Tivoli \& Zangrando, 2011). Esto también se sustenta con el registro arqueológico de zonas marginales como el archipiélago del Cabo de Hornos (Legoupil 1993-1994). Quizá el caso de Playa Larga (Yesner, 1990) corresponda a expresiones tempranas de uso más sostenido del espacio. Hay localidades etnográficas caracterizadas por la regularidad ocupacional, como ensenada San Martín, en la costa este de isla Hermite, cerca del cabo de Hornos, que era un "chosen haunt of the Yamana", sobre la base de la reiterada presencia de gente registrada por sucesivos navegantes (Chapman, 2010, pp. 75-76). Las observaciones de Fitz Roy indican que la costa noroeste de Navarino estaba entre las áreas más pobladas del territorio durante el primer tercio del siglo XIX, lugar que Chapman denomina "the Yamana Heartland" (Chapman, 2010, p. 138). Por supuesto sin tanta precisión temporal, ya desde los trabajos de Bird se observó la gran densidad de conchales del canal Beagle central en general, lo que se ha confirmado recientemente (Bird, 1938, 1946; Orquera \& Piana, 1999a; Mardones, 2016, 2019). Esto no significa que dicho nodo no se trasladara a otra localización con el paso del tiempo en sintonía con variados ritmos sociales y ecológicos. Todos estos casos pueden ser también útilmente explorados con el concepto de persistencia, que no implica necesariamente movilidad baja (Schlanger, 1992; Pope et al. 2018). Efectivamente, muchos sitios pudieron ser visitados múltiples veces por períodos relativamente cortos, como se ha sugerido, por ejemplo, para Ajej I (Piana et al. 2008, p. 114).

Son muchas veces mencionados los distintos distritos yaganes, regidos por principios de parentesco y con dialectos propios (Chapman, 2010, p. 366). Por supuesto, igual que ocurre con el caso de los haruwen selk'nam, se debe reconocer que este tipo de subdivisiones no constituye una segmentación fija, sino que seguramente ha sido variable a través del tiempo. Más allá de los efectos creados por variables grados de conocimiento por parte de los informantes, el contraste entre los 39 haruwen registrados por Gusinde para territorio selk'nam en el siglo XX y los 69 compilados por Chapman utilizando genealogías lo atestiguan (Gusinde, 1982 [1937], p. 397; Chapman, 1986). Particularmente relevante es que cuando Chapman compiló linajes dentro de cada haruwen observó que "los datos testimonian cierto fraccionamiento de los linajes y una inestabilidad en el número y en los límites de los haruwen" (Chapman, 1986, p. 39). Asimismo, las subdivisiones reconocidas por Aguilera y Tonko entre los kawésqar no corresponden a las informadas por Gusinde (Aguilera \& Tonko, 2011). En términos de geografía cultural, estas cambiantes situaciones significan que la capacidad de dar sentido territorial o de pertenencia depende de una enorme cantidad de variables que está constantemente redefiniendo las unidades espaciales reconocidas. Esto es totalmente concordante con observaciones acerca de la variable membresía de las agrupaciones de cazadoresrecolectores (Stanner, 1965; Binford, 2006).

Para tiempos históricos en la zona yagán se menciona la existencia de un territorio neutral, sobre la base de una información de Darwin. Esto ha sido considerado inexacto por varios autores (Chapman, 2010; Orquera \& Piana, 2015), pero Chapman termina sugiriendo la existencia de un territorio de esas características en "a zone along the Beagle Channel" (Chapman, 2010, pp. 181, 195-196). Este espacio se presentaría entre dos sociedades canoeras en Navarino, los yaganes hostiles a la tribu de Orondelico y la de Orondelico, al oeste (Chapman, 2010, p. 195). Asimismo, hay que mencionar la existencia de sectores tabú en territorio kawésqar, derivados de la configuración del terreno cuando éste se "apartaba de lo natural", 
al adquirir formas humanas o animales (Aguilera \& Tonko, 2011, p. 58). Usualmente los cazadoresrecolectores han considerado la existencia de espacios poco frecuentados por distintas razones, incluyendo espacios que evitaban (Collignon, 1996; Kay, 2007). Estas situaciones crean sectores neutrales.

\section{CONCLUSIONES}

Más allá de la riquísima información acerca de las divisiones dialectales $\mathrm{u}$ otras entre los distintos grupos reconocidos en tiempos históricos, resulta muy difícil aplicar -como muchas veces se hace- datos de fines del siglo XIX para discutir observaciones realizadas en el primer tercio de ese siglo. O sea, es muy difícil impugnar una observación etnográfica con otras realizadas más de 60 años después, como puede ocurrir con la cuestión de si las mujeres yaganes buceaban para obtener erizos o no (Cooper, 1917, p. 188). Esto es aún más evidente si consideramos que en el tiempo intermedio ocurre la masiva reorganización de las sociedades en su difícil contacto con el mundo occidental. Más allá del efecto de los mencionados contactos "fugaces", no puede minimizarse, por ejemplo, que en el primer cuarto del siglo XIX algunos canoeros inclusive habían viajado a Inglaterra con Fitz Roy y aprendido algo de inglés. Todo esto configura un panorama de tremenda dinámica cultural cuyos efectos aún no hemos terminado de ponderar. Por otra parte, la existencia de fluctuantes sectores del espacio considerados como tierra de nadie está muy bien registrada en sociedades de cazadores-recolectores (Stanner, 1965), al punto de constituir un ordenador geográfico importante, por lo que no debe llamar la atención su registro en la región.

Observaciones etnográficas en las islas Picton o Grévy (Martial, 2007 [1891]) indican el uso más o menos extendido de todo el territorio (Legoupil, 1993-1994; Legoupil \& Fontugne, 1997), lo que no significa uso continuo en tempo etnográfico. Las principales variaciones observadas se refieren precisamente a la intensidad ocupacional. Aceptando, dentro del esquema de Stuart (1983), que los canoeros no siempre arribaban al lugar planificado, se podían incluir usos no necesariamente prolongados de lugares muy pobres y poco atractivos, lo cual ha de ser válido a lo largo de todo el espacio archipelágico meridional. En otras palabras, hay condiciones bajo las cuales pudo fallar la planificación o ésta pudo flexibilizarse hasta sus límites llevando al uso de lugares poco óptimos. A veces éstos pueden ser resultado de la incertidumbre, por ejemplo, casos de canoeros que eran sorprendidos por cambios abruptos de las condiciones meteorológicas, que por ello debían permanecer en lugares no elegidos hasta que mejoraran las condiciones antes de continuar circulando. Asimismo, por razones naturales o culturales, parece adecuado aceptar que, en distintos momentos, ciertos lugares dejaron de frecuentarse.

Considerando toda esta información en tempo arqueológico, deberíamos esperar discontinuidades ocupacionales en alguna escala espacial aún no bien especificada, pero fuertemente dependiente de los patrones de movilidad.

\section{AGRADECIMIENTOS}

Este trabajo fue realizado bajo los auspicios de proyecto FONDECYT 1190984. Agradecemos a los dos evaluadores, a la revisión editorial y a todos los colegas que respondieron a nuestras consultas o generosamente compartieron información muchas veces inédita, entre ellos, Ken Ames, Juan Bautista Belardi, Karen Borrazzo, Miguel Chapanoff y Denis Chevallay.

\section{BIBLIOGRAFÍA}

Aguilera, O. E., \& Tonko, J. (2011). Guía Etnogeográfica del Parque Nacional Bernardo O'Higgins. InnovaChile CORFO-CEQUA-Comunidad Kawésqar de Puerto Edén.

Aguilera, O. E., \& Tonko, J. (2013). Relatos de viaje kawésqar. Ofqui editores.

Alfonso-Durruty, M. P., Vilar, M., \& Morello, F. (2016). Migration Waves and Genetic Drift in the Peopling of Fuego-Patagonia. Trabajo presentado al 85th Annual Meeting, American Association of Physical Anthropologists.

Alunni, D. V., Gutiérrez, M. A., \& Zangrando, A. F. (2017). Natural Accumulation and Distribution of Guanaco Bones in the Southernmost Tip of Tierra del Fuego (Argentina): Taphonomic Analysis and Archaeological 
Implications. Journal of Taphonomy, 15(1-3), 59-76.

Álvarez Saldivia, A. (2016). William Low. Lobero del fin del mundo. Editorial Atelí.

Álvarez, M., Pal, N., Salvatelli, L., Briz i Godino, I., Zangrando, A. F., Bazerque, A., De Angelis, H., \& Fiore, D. (2010). Tallando desde lo alto: un sitio de explotación de materiales líticos de los grupos cazadores-recolectores de la Isla Grande de Tierra del Fuego. Magallania, 38(1), 295-300.

Álvarez, M., Fiore, D., Tivoli, A., Salvatelli, L., Saletta, M. J., \& Briz, I. (2013). Variabilidad de actividades humanas en momentos recientes de la ocupación del canal Beagle: El caso de Lanashuaia XXI. En A. F. Zangrando, R. Barberena, A. Gil, G. Neme, M. Giardina, L. Luna, C. Otaola, S. Paulides, L. Salgán \& A. Tivoli (Eds.), Tendencias teórico-metodológicas y casos de estudio en la arqueología de Patagonia (pp. 559-568). Museo de Historia Natural de San Rafael.

Ames, K. (2002). Going by Boat: The Forager-Collector Continuum at Sea. En B. Fitzhugh \& J. Habu (Eds.), Beyond Foraging and Collecting: Evolutionary Change in Hunter-Gatherer Settlement Systems (pp. 17-50). Kluwer/Plenum Press.

Andrade, S. G. (1991). Geomorfología costera, antecedentes oceanográficos físicos de la región de Magallanes. Chile (48 $\left.{ }^{\circ}-56^{\circ} \mathrm{S}\right)$. Anales del Instituto de la Patagonia, Serie Ciencias Naturales, 20, 135-151.

Aracena, C., Kilian, R., Lange, C. B., Bertrand, S., Lamy, F., Arz, H. W., De Pol-Holz, R., Baeza, O., Pantoja, S., \& Kissel, C. (2015). Holocene variations in productivity associated with changes in glacier activity and freshwater flux in the central basin of the Strait of Magellan. Palaeogeography, Palaeoclimatology, Palaeoecology, 436, 112-122.

Arroyo, M. T. K., Donoso, C., Murúa, R. E., Pisano, E. E., Schlatter, R. P., \& Serey, I. A. (1996). Toward an Ecologically Sustainable Forestry Project. Concepts, analysis and recommendations. Departamento de Investigación y Desarrollo, Universidad de Chile.

Arroyo, M. T. K., Minoc, M., Pliscoff, P., \& Arroyo-Kalin, M. (2005). The Magellanic moorland. En L. H. Fraser \& P. A. Keddy (Eds.), The World's Largest Wetlands Ecology and Conservation (pp. 424-445). Cambridge University Press.

Arthur, W. B. (2009). The Nature of Technology. New York: Free Press.

Aspillaga, E., \& Ocampo, C. (1996). Restos óseos humanos de la Isla Karukinka (Seno Almirantazgo, Tierra del Fuego). Informe preliminar. Anales del Instituto de la
Patagonia, Serie Ciencias Humanas, 24, 153-161.

Aspillaga, E., Ocampo, C., \& Rivas, P. (1999). Restos óseos humanos de contextos arqueológicos del área de Navarino: indicadores de estilo de vida en indígenas canoeros. Anales del Instituto de la Patagonia, Serie Ciencias Humanas, 26, 123-136.

Banks, W. E. (2017). Improving eco-cultural niche estimations: the potential of archaeological faunal remains for taking biotic interactions into consideration. En J-P. Brugal (Ed.), TaphonomieS (pp. 498-507). Éditions des archives contemporaines.

Berihuete Azorín, M. (2009). El papel de los recursos vegetales no leñosos en las economías cazadoras-recolectoras: Propuesta para el estudio de su gestión: El caso de Tierra del Fuego, Argentina. Facultat de Lletres, Universitàt Autònoma de Barcelona.

Berihuete Azorín, M. (2014). Las plantas en las economías fueguinas: una perspectiva etnoarqueológica. En J. Oría \& A. M. Tivoli (Eds.), Cazadores de mar y tierra: estudios recientes en arqueología fueguina (pp. 389408). Museo del Fin del Mundo.

Binford, L. R. (1982). The Archaeology of Place. Journal of Anthropological Archaeology, 1, 5-31.

Binford, L. R. (1983a). Long-term Land-use Patterning: Some Implications for Archaeology. In L. R. Binford (Ed.), Working at Archaeology (pp. 379-386). Academic Press.

Binford, L. R. (1983b). In Pursuit of the Past: Decoding the Archaeological Record. Thames and Hudson.

Binford, L. R. (1987). Searching for Camps and Missing the Evidence? Another Look at the Lower Paleolithic. In O. Soffer (Ed.), The Pleistocene Old World (pp. 17-31). Plenum Press.

Binford, L. R. (1991). When the going gets tough, the tough get going: Nunamiut local groups, camping patterns and economic organisation. In C. Gamble \& W. A. Boisnier (Eds.), Ethnoarchaeological Approaches to Mobile Campsites (pp. 25-138). International Monographs in Prehistory.

Binford, L. R. (2006). Bands as Characteristic of "Mobile Hunter-gatherers" may exist only in the History of Anthropology. In F. Sellet, R. Greaves \& P-L. Yu (Eds.), Archaeology and Ethnoarchaeology of Mobility (pp. 3-22). University of Florida Press.

Bird, J. (1932). Tierra del Fuego 1932-1933. Letters 1934-37. AMNH, Ms.

Bird, J. (1938). Antiquity and migrations of the early inhabitants of Patagonia. The Geographical Review, XXVIII(2), 250-275. 
Bird, J. (1946). The Archaeology of Patagonia. In J. H. Steward (Ed.), Handbook of South American Indians (Vol. 1, pp. 17-24). Bureau of American Ethnology Bulletin 143, Smithsonian Institution.

Bishop, C. A. (1978). Cultural and biological adaptations to deprivation: The northern Ojibwa case. In C. D. Laughlin \& I. A. Brady (Eds.), Extinction and survival in human populations (pp. 208-230). Columbia University Press.

Borrazzo, K. (2012). Raw material availability, flaking quality and hunter-gatherer decision making in Northern Tierra del Fuego Island (Argentina). Journal of Archaeological Science, 39, 2643-2654.

Borrazzo, K., Borrero, L. A., \& Pallo, C. (2019). Exploring lithic transport in Tierra del Fuego (Southern South America). Journal of Archaeological Science: Reports, 24, 220-230.

Borrero, L. A. (1984). Reseña de "Los Indios de Tierra del Fuego", Tomo I. Los Selk'nam, de Martín Gusinde. Scripta Ethnologica 8, 169-173.

Borrero, L. A. (1991). Los 'modelos de situaciones excepcionales' y el estudio de las sociedades de cazadores y recolectores. Comechingonia, 8(7), 107127.

Borrero, L. A. (2002). El Poblamiento de la Patagonia. Toldos, milodones y volcanes. Editorial Emecé.

Borrero, L. A. (2011). Los cazadores orientales de Tierra del Fuego. En A.F. Zangrando, M. Vázquez \& A. Tessone (Eds.), Los cazadores-recolectores del extremo oriental fueguino Arqueología de Península Mitre e Isla de los Estados (pp. 287-298). Sociedad Argentina de Antropología.

Borrero, L. A., \& Borrazzo, K. (2011). La geografía cultural del sudoeste de Patagonia continental. En L.A. Borrero \& K. Borrazzo (Eds.), Bosques, montañas y cazadores. Investigaciones Arqueológicas en Patagonia Meridional (pp. 7-36). CONICET-IMHICIHU, Buenos Aires.

Borrero, L. A., Martin, F. M., \& Barberena, R. (2011). Visits, "Fuegians," and Information Networks. In R. Whallon, W.A. Lovis \& R. K. Hitchcock (Eds.), Information and its Role in Hunter-Gatherer Bands (pp. 249-265). The Cotsen Institute of Archaeology at UCLA.

Bown, F., Rivera, A., Zenteno, P., Bravo, C., \& Cawkwell, F. (2014). First glacier inventory and recent glacier variation on Isla Grande de Tierra del Fuego and adjacent islands in Southern Chile. In J. S. Kargel, G. J. Leonard, M.P. Bishop, A. Kaab \& B. Raup (Eds.), Global Land Ice Measurements from Space (pp. 661-
674). Springer-Praxis.

Breivik, H. M., Bjerck, H. B., Zangrando, A. F. J., \& Piana, E. L. (2016). On the applicability of environmental and ethnographic reference frames: an example from the high-latitude seascapes of Norway and Tierra del Fuego. In H. B. Bjerck, H. M. Breivik, S. E. Fretheim, E. L. Piana, B. Skar, A. M. Tivoli \& A. F. J. Zangrando (Eds.), Marine Ventures (pp. 75-94). Equinox.

Bridges, E. L. (1951). The Uttermost Part of the Earth. Hodder and Stoughton.

Bridges, E. L. (1952). El último confín de la tierra. El Ateneo. Bridges, T. (2001). Los indios del último confín. Zagier \& Urruty Publications.

Briz Godino, I., Álvarez, M., Balbo, A., Zurro, D., Madella, M., Villagrán, X., \& French, C. (2011). Towards highresolution shell midden archaeology: Experimental and ethnoarchaeology in Tierra del Fuego (Argentina). Quaternary International, 239, 125-134.

Byron, J. (1996 [1768]). Naufragio en las costas patagónicas. Ediciones del Sol.

Cane, S. (1990). Desert demography: A case Study of PreContact Aboriginal Densities. In B. Meehan \& N. White (Eds.), Hunter-Gatherer Demography. Past and present. Oceania Monograph, 39, 149-159. University of Sydney.

Caniupán, M., Lamy, F., Lange, C. B., Kaiser, J., Kilian, R., Arz, H. W., León, T., Mollenhauer, G., Sandoval, S., De Pol-Holz, R., Pantoja, S., Wellner, J., \& Tiedemann, R. (2014). Holocene sea-surface temperature variability in the Chilean fjord region. Quaternary Research, 82, 342-353.

Cárdenas, C. A., \& Montiel, A. (2016). Coexistence in Cold Waters: Animal Forests in Seaweed-Dominated Habitats in Southern High Latitudes. In S. Rossi (Ed.) Marine Animal Forests (pp. 1-20). Springer International.

Carre, M., Sachs, J. P., Purca, S., Schauer, A. J., Braconnot, P., Falcon, R. A., Julien, M., \& Lavallee, D. (2014). Holocene history of ENSO variance and asymmetry in the eastern tropical Pacific. Science, 345(6200), 10451048. https://doi.org/10.1126/science. 1252220

Caruso Fermé, L., Álvarez, M., \& Vázquez, M. (2011). Análisis arqueobotánico de piezas de madera del extremo austral americano. Magallania, 39(1), 221-240.

Caruso Fermé, L., \& Zangrando, A. F. J. (2018). Anthracological analyses of fuel wood used by hunter-gatherers in the south coast of Tierra del Fuego during the late Holocene. Archaeological and Anthropological Sciences, https://doi.org/10.1007/s12520-018 0652-4 
Chapman, A. (1986). Los Selk'nam. La vida de los onas. Emecé Editores.

Chapman, A. (2010). European Encounters with the Yamana People of Cape Horn, Before and After Darwin. Cambridge University Press.

Charlin, J., Cardillo, M., \& Borrazzo, K. (2014). Spatial patterns in Late Holocene lithic projectile point technology of Tierra del Fuego: assessing size and shape changes. World Archaeology, 46(1), 78-100.

Charlin, J., Augustat, C., \& Urban, C. (2016). Metrical variability in ethnographic arrows from southernmost Patagonia: Comparing collections from Tierra del Fuego at European museums. Journal of Anthropological Archaeology, 41(1), 313-326.

Chase, O. (2000). Narrative of the Most Extraordinary and Disastrous Shipwreck of the Whale-ship Essex. In T. Philbrick \& N. Philbrick (Eds.), The Loss of the Ship Essex Sunk by a Whale. First-Person Accounts, (pp. 1-231). Penguin Books.

Christensen, M. (2016). La industria ósea de los cazadoresrecolectores: el caso de los nómades marinos de Patagonia y Tierra del Fuego Chile. Ediciones Universidad de Magallanes.

Collignon, B. (1996). Les Inuit. Ce Qu'ils savant du territoire. L'Harmattan.

Cooper, J. M. (1917). Analytical and critical bibliography of the tribes of Tierra del Fuego and adjacent territory. Bureau of American Ethnology. Bulletin 63. Smithsonian Institution, Washington D. C.

Coronato, A., Mazzoni, E., Vázquez, M., \& Coronato, F. (2017). Patagonia. Una sintesis de su geografía física. UNPA.

Darwin, C. (1906 [1839]). Journal of Researches into the Geology \& Natural History of the Various Countries Visited During the Voyage of the Beagle Round the World. Dent \& Sons.

De Beaune, S. A. (2011). Le processus de l'invention: Approche cognitive. En R. Treuil (Ed.), L'Archéologie cognitive (pp. 75-90). Paris: Éditions de la Maison des sciences de l'homme.

De la Fuente, C., Galimany, J., Kemp, B. M., Judd, K., Reyes, O., \& Moraga, M. (2015). Ancient marine huntergatherers from Patagonia and Tierra del Fuego: Diversity and differentiation using uniparentally inherited genetic markers. American Journal of Physical Anthropology, 158(4), 719-729.

D'Errico, F., Banksa, W. E., Warren, D.L., Sgubin, G., van Niekerk, K., Henshilwood, C., Daniau, A-L., \& Sánchez Goñi, M. F. (2017). Identifying early modern human ecological niche expansions and associated cultural dynamics in the South African Middle Stone Age. Proceedings of the National Academy of Sciences, 114(30), 7869-7876.

Derex, M., \& Boyd, R. (2016). Partial connectivity increases cultural accumulation within groups. Proceedings of the National Academy of Sciences 113 (11), 29822987.

Díaz Lomovasky, A. (2018). Análisis arqueofaunístico de mamíferos y aves de los sitios FP1, SA1, SM2 y SM3, Región de Magallanes y la Antártica Chilena. Departamento de Antropología, Facultad de Ciencias Sociales, Universidad de Chile.

Duplessis, R. S. (2003 [1699-1701]). Périple de Beauchesne à la Terre de Feu (1698-1701), une expédition mandatée par Louis XIV. Paris: Éd. Transboréal.

Emperaire, J. (1963 [1955]). Los nómades del Mar. Santiago: Ediciones de la Universidad de Chile.

Enloe, J. (2010). Refitting Bones: Negative Evidence, Site Structure and Social Organization. Lithic Technology, 34(2), 23-29.

Fernández, M., Ponce, J. F., Zangrando, F. J., Borromei, A. M., Musotto, L. L., Alunni, D., \& Vázquez, M. (2018). Relationships between terrestrial animal exploitation, marine hunter-gatherers and palaeoenvironmental conditions during the Middle-Late Holocene in the Beagle Channel region. Quaternary International, doi.org/10.1016/j.quaint.2018.05.032

Figuerero Torres, M.J., \& Mengoni Goñalons, G. (1986). Excavaciones arqueológicas en isla El Salmón (Parque Nacional de Tierra del Fuego). PREP Informes de Investigación, 4, 1-94.

Fiore, D. (2011). Art in time. Diachronic rates of change in the decoration of bone artefacts from the Beagle Channel region (Tierra del Fuego, Southern South America). Journal of Anthropological Archaeology, 30(4), 484501.

Fiore, D., Maier, M., Parera, S. D., Orquera, L., \& Piana, E. (2008). Chemical analyses of the earliest pigment residues from the uttermost part of the planet (Beagle Channel region, Tierra del Fuego, Southern South America). Journal of Archaeological Science, 35, 3047-3056.

Fitz Roy, R. (1839). Narrative of the surveying voyages of His Majesty's Ships Adventure and Beagle between the years 1826 and 1836, describing their examination of the southern shores of South America, and the Beagle's circumnavigation of the globe. Proceedings of the second expedition, 1831-36, under the command of Captain Robert Fitz-Roy, R.N. Henry 
Colburn, London.

Fitz Roy, R. (2016 [1839]). Los viajes del Beagle. Informes de la segunda expedición (1831-1836). Buenos Aires: EUDEBA.

Försterra, G., Häussermann, V., \& Laudien, J. (2016). Animal Forests in the Chilean Fjords: Discoveries, Perspectives and Threats in Shallow and Deep Waters. En S. Rossi (Ed.), Marine Animal Forests (pp. 1-36). Springer International Publishing.

Gamble, C., \& Gaudzinski, S. (2007). Bones and powerful individuals: faunal case studies from the Arctic and the European Middle Palaeolithic. En C. Gamble \& M. Porr (Eds.), The hominid individual in context. Archaeological investigations of Lower and Middle Palaeolithic landscapes, locales and artefacts (pp. 154-175). Routledge.

Gawronski, J. (1999). La Exploración y los Hallazgos Arqueológicos. En P. Floore, J. Gawronski, O. Hefting \& J. J. Zeeberg (Eds.), Holandeses en el Estrecho de Magallanes (pp. 45-52). Stichting Olivier Van Noort y el Instituut voor Pre- en Protohistorische Archeologie, Universiteit van Amsterdam.

Gibbons, J., Gazitúa, F., \& Venegas, C. (2000). Cetáceos en el estrecho de Magallanes y senos Otway, Skyring y Almirantazgo. Anales del Instituto de la Patagonia, Serie Ciencias Naturales, 28, 107-118.

Grove, M. (2018). Hunter-gatherers adjust mobility to maintain contact under climatic variation. Journal of Archaeological Science: Reports 19, 588-595.

Gusinde, M. (1982 [1937]). Los indios de Tierra del Fuego. Tomo I (I) Los Selknam. CAEA-CONICET, Buenos Aires.

Gusinde, M. (1986a [1937]). Los indios de Tierra del Fuego. Tomo II (III). Los Yámana. CAEA-CONICET, Buenos Aires.

Gusinde, M. (1986b [1937]). Los indios de Tierra del Fuego. Tomo II (I). Los Yámana. CAEA-CONICET, Buenos Aires.

Gusinde, M. (1991a [1974]). Los indios de Tierra del Fuego. Tomo III (I). Los Halakwulup. CAEA-CONICET, Buenos Aires.

Higgs, E. S., \& Vita-Finzi, C. (1972). Prehistoric economies: a territorial approach. En E. S. Higgs (Ed.), Papers in Economic Prehistory (pp. 27-36). Cambridge: Cambridge University Press.

Hyades, P., \& Deniker, J. (2007 [1891]). Los indígenas según Hyades \& Deniker. En D. Legoupil \& A. Prieto (Eds.), Etnografía de los indios Yaghan en la Misión Científica del Cabo de Hornos 1882-1883 (pp. 69-
329). UMAG-Instituto Francés de Estudios Andinos, Punta Arenas.

Inizan, M. L., Reduron, M., Roche, H., \& Tixier, J. (1995). Technologie de la Pierre Taillée (Vol. 4). CREPCNRS. Meudon.

Jochim, M. (1981). Strategies for Survival. Cultural Behavior in an Ecological Context. Academic Press.

Kay, C. E. (2007). Were Native People Keystone Predators? A Continuous-Time Analysis of Wildlife Observations Made by Lewis and Clark in 1804-1806. The Canadian Field-Naturalist, 121(1), 1-18.

Koch, J. (2015). Little Ice Age and recent glacier advances in the Cordillera Darwin, Tierra del Fuego, Chile. Anales del Instituto de la Patagonia, 43(1), 127-136.

Kochi, S. (2017). Paleodietas en cazadores-recolectores del canal Beagle durante el Holoceno tardío. Intersecciones en Antropología, 18, 329-339.

Kochi, S., Pérez, S. A., Tessone, A., Ugan, A., Tafuri, M. A., Nye, J., Tivoli, A. M., \& Zangrando, A. F. (2017). $\delta^{13} \mathrm{C}$ and $\delta^{15} \mathrm{~N}$ variations in terrestrial and marine foodwebs of Beagle Channel in the Holocene. Implications for Human Paleodietary Reconstructions. Journal of Archaeological Science. Reports, 18, 696-707.

Koppers, W. (1977 [1924]). Entre los fueguinos. Ediciones Universidad de Magallanes.

Lee, R. B. (1979). The !Kung San: Men, Women and Work in a Foraging Society. Cambridge University Press. // Correcto: se escribe asi///

Lefèvre, C. (1993-1994). Las aves en los yacimientos del archipiélago del Cabo de Hornos y del seno Grandi. Anales del Instituto de la Patagonia, 22, 123-136.

Legoupil, D. (1985-1986). Los indios de los archipiélagos de la Patagonia, un caso de adaptación a un ambiente adverso. Anales del Instituto de la Patagonia, 16, 45-52.

Legoupil, D. (1993-1994). El archipiélago del cabo de Hornos y la costa sur de la isla Navarino: poblamiento y modelos económicos. Anales del Instituto de la Patagonia, 22, 101-121.

Legoupil, D., \& Fontugne, M. (1997). El Poblamiento Marítimo en los Archipiélagos de Patagonia: Núcleos Antiguos y Dispersión Reciente. Anales del Instituto de la Patagonia, 25, 75-87.

Lemonnier, P. (1983). L'Etude des systemes techniques, une urgence en Technologie Culturelle. Techniques et Culture, 2, 11-34.

Lemonnier, P. (1991). De la culture matérielle à la culture? Ethnologie des techniques et Préhistoire. En APDCA (Ed.), 25 ans d'études technologiques en Préhistoire 
(pp. 15-20). XIe Rencontres Internationales d'Archéologie et d'Histoire d'Antibes, Juan-les-Pins.

Lupo, K. D., Fancher, J. M., \& Schmitt, D. N. (2013). The Taphonomy of Resource Intensification: Zooarchaeological Implications of Resource Scarcity Among Bofi and Aka Forest Foragers. Journal of Archaeological Method and Theory, 20, 420-447.

Maerky, G. (2018). Projectile shafts of recent hunter-gatherer's Subarctic and Sub-Antartic societies in America. Book of Abstracts. 18th UISPP World Congress, Paris.

Mardones, J. (2016). Microambientes y zooarqueología en la costa sur del canal Beagle. Un enfoque espacial sobre la explotación de recursos. Tesis para optar al grado de arqueóloga, Facultad de Ciencias Sociales, Universidad de Chile.

Mardones, J. (2019). Subsistencia en isla Navarino. Un estudio exploratorio sobre la variabilidad espacial en la explotación de recursos al sur del canal Beagle, Chile. Magallania, 47(1), 145-157.

Martial, L. F. (2005 [1888]). Misión al Cabo de Hornos. Zagier \& Urruty Publications.

Martial, L. F. (2007 [1891]). Los indígenas según Martial. En D. Legoupil \& A. Prieto (Eds.), Etnografía de los indios Yaghan en la Misión Científica del Cabo de Hornos 1882-1883 (pp. 18-68). Ediciones Universidad de Magallanes-Instituto Francés de Estudios Andinos, Punta Arenas.

Martinic, M. (1973). Actividad lobera y ballenera en litorales y aguas de Magallanes y Antártica, 1868-1916. Revista de Estudios del Pacífico, 7, 7-26.

Martinic, M. (2011). El occidente fueguino. Todavía una incógnita. Gasco Magallanes.

Matano, R. P., \& Palma, E. D. (2008). On the upwelling of downwelling currents. Journal of Physical Oceanography 38(11), 2482-2500.

Mayorga, M. (2017). Actividad lobera temprana en la Patagonia Oriental: caza de mamíferos marinos. Rivar, 4(11), 3151.

Mayorga, M. (2018). Loberos yankees: Encuentros/ desencuentros en torno a la Tierra del Fuego y Patagonia. Revista Estudios Hemisféricos y Polares, 9(4), 28-44.

Meehan, B. (1982). Shell Bed to Shell Midden. Australian Institute of Aboriginal Studies, Canberra.

Minc, L. D. (1986). Scarcity and Survival: The Role of Oral Tradition in Mediating Subsistence Crises. Journal of Anthropological Archaeology, 5(1), 39-113.

Moore, D. M. (1983). Flora of Tierra del Fuego. Nelson, Oswestry.
Moraga, M., de Saint Pierre, M., Torres, F., \& Ríos, J. (2010). Vínculos de parentesco por vía materna entre los últimos descendientes de la etnia kawésqar y algunos entierros en los canales patagónicos: evidencias desde el estudio de los linajes mitocondriales. Magallania, 38(2), 103-114.

Morello, F. (2016). L'industrie lithique de part et d'autre du Détroit de Magellan, en Patagonie et Terre de Feu: dynamiques techno-culturelles du peuplement durant l'Holocène. Tesis de Doctorado. L'Université de Paris I Panthéon-Sorbonne, Paris.

Morello, F., Alfonso-Durruty, M., Amorosi, T., Martínez, I., Massone, M., Martin, F., Borrero, L. A., Christensen, M., Torres, J., Sierpe, V., Reyes, O., \& San Román, M. (2018). Junius Bird's collections from sites rockshelter 1, 2 and 3 (Beagle Channel, Patagonia, Chile). Trabajo presentado al 83rd Meeting of the Society for American Archaeology, Washington D.C.

Muñoz, C., Cordero, R., \& Artigas, D. (2016). El sitio Alero Picton 1: Nuevo registro de arte rupestre para los canales fueguinos. Magallania, 44(2), 225-231.

Obelic, B., Álvarez, A., Argullós, J., \& Piana, E. (1998). Determination of water paleotemperature in the Beagle Channel (Argentina) during the last 6000 yr through stable isotope composition of Mytilus edulis shells. Quaternary of South America and Antarctic Peninsula, 11, 47-71.

Ocampo C., \& Rivas, P. (2000). Nuevos fechados ${ }^{14} \mathrm{C}$ de la costa norte de la isla Navarino, costa sur del Canal Beagle, Provincia Antártica Chilena, Región de Magallanes. Anales del Instituto de la Patagonia, 28, 197-214.

Odling-Smee, F., Laland, J., \& Feldman, M. (2003). Niche Construction. The Neglected Process in Evolution. Princeton University Press.

Orquera, L.A. (2002). The Late-Nineteenth-Century Crisis in the Survival of the Magellan-Fuegian Littoral Natives. En C. Briones \& J. L. Lanata (Eds.), Archaeological and Anthropological Perspectives on the Native Peoples of Pampa, Patagonia, and Tierra del Fuego to the Nineteenth Century (pp. 141-158). Bergin \& Garvey, Westport.

Orquera, L. A., \& Piana, E. L. (1996). El sitio Shamakush I (Tierra del Fuego, República Argentina). Relaciones de la Sociedad Argentina de Antropología, 21, 215265.

Orquera, L. A., \& Piana, E. L. (1999a). Arqueología de la región del canal Beagle (Tierra del Fuego, República Argentina). Sociedad Argentina de Antropología.

Orquera, L. A., \& Piana, E. L. (1999b). La vida material y 
social de los Yámana. EUDEBA.

Orquera, L. A., \& Piana, E. L. (2009). Sea Nomads of the Beagle Channel in Southernmost South America: Over Six Thousand Years of Coastal Adaptation and Stability. The Journal of Island and Coastal Archaeology, 4(1), 61-81.

Orquera, L. A., \& Piana, E. L. (2015). La vida material y social de los Yámana. $2^{\circ}$ ed., Ediciones Monte Olivia.

Orquera, L. A., Piana, E. L., Fiore, D., \& Zangrando, A. F. (2012). 10.000 años de fuegos. Arqueología y etnografía del fin del mundo. Editorial Dunken.

Ortiz Troncoso, O. (1972). Una nota sobre un yacimiento arqueológico en el archipiélago del Cabo de Hornos. Anales del Instituto de la Patagonia, 3(1-2), 83-85.

Ozán, I., \& Pallo, C. (2019). Past human populations and landscapes in the Fuegian Archipelago, southernmost South America. Quaternary Research, 1-19. https:// doi.org/10.1017/qua.2018.157

Pallo, M. C. (2011). Condicionamiento de la dinámica ambiental en las decisiones humanas sobre asentamiento $y$ circulación a lo largo del Estrecho de Magallanes durante el Holoceno Tardío. Magallania, 39(2), 177192.

Piana, E. L. (2005). Cetaceans and humans beings at the uttermost part of America: a lasting relationship in Tierra del Fuego. In G. Monks (Ed.), The Exploitation and Cultural Importance of Sea Mammals (pp. 121137). Oxbow Books.

Piana, E. L., \& Canale, G. (1993-1994). "Túnel II: un yacimiento de la Fase Reciente del Canal Beagle". Relaciones de la Sociedad Argentina de Antropología, XIX, 363-389.

Piana, E. L., \& Orquera, L. A. (1998). Canoe fuegine: etnografia storica $e$ archeologia. L'essemplare del Museo L'. Pigorini. Bulletino di Paletnologia Italiana, 89, Nuova Serie VII, 397-445.

Piana, E. L., Estévez, J., \& Vila Mitja, A. (2000). Lanashuaia: un sitio de canoeros del siglo pasado en la costa norte del Canal Beagle. En Desde el País de los Gigantes. Perspectivas Arqueológicas de la Patagonia (pp. 455469). Universidad Nacional de la Patagonia Austral.

Piana, E. L., Tessone, A., \& Zangrando, A. (2006). Contextos mortuorios en la región del Canal Beagle: del hallazgo fortuito a la búsqueda sistemática. Magallania, 34(1), 87-101.

Piana, E. L., Vázquez, M., \& Álvarez, M. (2008). Nuevos resultados del estudio del sitio Ajej I: un aporte a la variabilidad de estrategias de los canoeros fueguinos. Runa, 29, 101-121.

Pigeot, N. (1991). Reflexions sur l'histoire technique de
l'Homme: de l'evolution cognitive a l'evolution culturelle. Paléo, 3, 167-200.

Piqué, R. (1999). Producción y uso del combustible vegetal: una evaluación arqueológica. Treballs d'Etnoarqueologia 3. CSIC-UAB

Pisano, E. (1977). Fitogeografía de Fuego-Patagonia chilena. I. Comunidades vegetales entre las latitudes $52^{\circ}$ y $56^{\circ}$ S. Anales del Instituto de la Patagonia, 8, 121-250.

Politis, G. (2007). Nukak. Ethnoarchaeology of an Amazon People. Left Coast Press.

Ponce, J. F., Borromei, A. M., \& Rabassa, J. O. (2011). Evolución del paisaje y de la vegetación durante el Cenozoico tardío en el extremo sudeste del archipiélago Fueguino y Canal Beagle. En A.F. Zangrando, M. Vázquez \& A. Tessone (Eds.), Los cazadores-recolectores del extremo oriental fueguino. Arqueología de península Mitre e Isla de los Estados (pp. 51-63). Sociedad Argentina de Antropología.

Pope, M., McNann, J., \& Gamble, C. (Eds.) (2018). Crossing the Human Threshold. Dynamic Transformation and Persistent Places during the Middle Pleistocene. Routledge.

Prieto, M. R., \& Herrera, R. (1999). Austral Climate and Glaciers in the 16th Century through the Observations of the Spanish Navigators. Quaternary of South America and Antarctic Peninsula, 11, 153-179.

Promis, A., Cruz, G., Reif, A., \& Gärtner, S. (2008). Nothofagus betuloides (Mirb.) Oerst 1871 (Fagales: Nothofagaceae) Forests in southern Patagonia and Tierra del Fuego. Anales Instituto Patagonia, 36, 53-68.

Quiroz, D., Carreño, G. \& De La Fuente, P. (2016). El procesamiento de ballenas varadas en las costas de la patagonia occidental la propósito de un grabado de mediados del siglo xix]. Magallania, 44(1), 57-72.

Ratto, N. (2003). Estrategias de caza y propiedades del registro en la Puna de Chaschuil (Depto Tinogasta, Catamarca, Argentina). Tesis Doctoral, Universidad de Buenos Aires.

Ratto, N., \& Marconetto, B. (2012). Proyectiles en Acción, 20 Años Después.... Diseños en la fabricación de astiles fueguinos de colecciones etnográficas. En J. Martínez \& D. Bozzuto (Eds.), Armas Prehispánicas, Múltiples Enfoques para su Estudio en Sudamérica (pp. 135150). Ediciones FHN Félix de Azara.

Riso Patrón, L. (1924). Diccionario Jeográfico de Chile. Imprenta Universitaria.

San Román, M. (2014). Sea-Level Changes and Coastal Peopling in Southernmost Pacific South America: Marine Hunters from Patagonia. In C. Smith. (Ed.), 
Encyclopedia of Global Archaeology (pp. 65156525). Springer-Verlag, New York.

San Román, M. (2016). Stratégies économiques et sociales des chasseurs marins de Patagonie: Archéozoologie des sites anciens du détroit de Magellan et des mers intérieures. Tesis de Doctorado. L'Université de Paris I Panthéon-Sorbonne, Paris.

San Román, M., \& Morello, F. (2001). Canal Maule: nuevos antecedentes sobre prácticas funerarias en el archipiélago fueguino. Anales del Instituto de la Patagonia, 29, 149-161.

Santiago, F., \& Vázquez, M. (2009). Dietas promediadas: explorando el registro zooarqueológico supraregional en Tierra del Fuego. Revista del Museo de Antropología, 5, 225-238.

Scheinsohn, V. (2011). El trabajo del hueso en el fin del mundo: tecnología ósea en Bahía Valentín. En A. F. Zangrando, M. Vázquez \& A. Tessone (Eds.), Los cazadoresrecolectores del extremo oriental fueguino. Arqueología de península Mitre e Isla de los Estados (pp. 271-286). Sociedad Argentina de Antropología.

Schlanger, S. H. (1992). Recognizing Persistent Places in Anasazi Settlement Systems. In J. Rossignol \& L. A. Wandsnider (Eds.), Space, Time, and Archaeological Landscapes (pp. 91-112). Springer

Simberloff, D. (1988). The Contribution of Population and Community Biology to Conservation Science. Annual Review of Ecology and Systematics, 19, 473-511.

Skottsberg, C. (1913). Observations on the natives of the patagonian channel region. American Anthropologist, 15, 578-616.

Skottsberg, C. (2004 [1911]). La Patagonia salvaje. Zagier \& Urruty Publications.

Smith, B. (1979). Wind, Waves and Whales. Cape Horn by Kayak. Expedition, 9(4), 10-14.

Solari, M. E. (1993-1994). Estudio antracológico del archipiélago del Cabo de Hornos y Seno Grandi. Anales del Instituto de la Patagonia, 22, 137-148.

Speth, J. D. (2010). The Paleoanthropology and Archaeology of Big-Game Hunting. Protein, Fat, or Politics? Springer, New York.

Stambuk, P. (2011 [1986]). Rosa Yagán. Lakutaia le kipa. Pehuén Editores.

Stanner, W. E. H. (1965). Aboriginal territorial organization: Estate, range, domain and regime. Oceania, 36(1), 1-26.

Stuart, D. E. (1983). Subsistencia Yahgan y patrones de movilidad en canoas. Arqueología Contemporánea, 1(1), 20-27.
Suby, J. A., Luna, L., Aranda, C., \& Flensborg, G. (2017). First approximation to paleodemography through ageat-death profiles in hunter-gatherers from Southern Patagonia during middle-late Holocene. Quaternary International, 438, 174-188.

Tafuri, M. A., Zangrando, A. F. J., Tessone, A., Kochi, S., Moggi Cecchi, J., Di Vincenzo, F.,.. Manzi, G. (2017). Dietary resilience among hunter-gatherers of Tierra del Fuego: Isotopic evidence in a diachronic perspective. PLoS ONE 12(4): e0175594. https://doi. org/10.1371/journal. pone.0175594

Terradas, X., Vila, A., Clemente, I., \& Mansur, M. E. (1999). Ethno-neglect or the Constradiction Between Ethnohistorical Sources and the Archaeological Record: The Case of Stone Tools from the Yamana (Tierra del Fuego, Argentina). Urgeschichtliche Materialhefte, 14, 103-115.

Tessone, A., Guichón, R. A., Suby, J., \& Kozameh, L. F. (2011). Bioarqueología de Península Mitre. En A. F. Zangrando, M. Vázquez \& A. Tessone (Eds.), Los cazadores-recolectores del extremo oriental fueguino. Arqueología de península Mitre e Isla de los Estados (pp. 231-270). Sociedad Argentina de Antropología, Buenos Aires.

Tivoli, A. M. (2010). Las aves en la organización socioeconómica de cazadores-recolectorespescadores del extremo sur sudamericano. Tesis Doctoral Inédita, Universidad de Buenos Aires.

Tivoli, A., \& Zangrando, A. F. (2011). Subsistence variations and landscape use among maritime hunter-gatherers. A zooarchaeological analysis from the Beagle Channel (Tierra del Fuego, Argentina). Journal of Archaeological Science, 38(5), 1148-1156.

Tonko, J. (2019). Entrevista. UMAG TV Páginas de Nuestra Historia, Punta Arenas.

Tuhkanen, S., Kuokka, I., Hyvoenen, J., Streenroos, S., \& Niermelä, J. (1989-1990). Tierra del Fuego as a target for biogeographical research in the past and present. Anales del Instituto de la Patagonia, 19, 1-107.

Vázquez, M., \& Zangrando, F. (2017). Estructuras de pesca en el canal Beagle. Magallania, 45(1), 101-122.

Venegas, C., \& Sielfeld, W. (1979). Antecedentes para la determinación de un nuevo distrito zoogeográfico en el litoral exterior de Magallanes. Anales del Instituto de la Patagonia, 10, 201-208.

Vidal, H. J., \& Winograd, A. J. (1986). El factor humano: modificaciones antropogénicas en la paleoecología de otáridos en Tierra del Fuego. Resumos do Reuniao de Trabalho de Especialistas em Mamiferos Aquaticos 
da America do Sul 2, (pp. 96-99). FBCN.

Weddell, J. (2006 [1825]). Un viaje hacia el Polo Sur realizado en los años 1822-1824. EUDEBA.

Whallon, R. (2011). An Introduction to Information and its Role in Hunter-Gatherer Band-Level Societies. In R. Whallon, W.A. Lovis \& R. K. Hitchcock (Eds.), Information and its Role in Hunter-Gatherer Bands (pp. 1-28). The Cotsen Institute of Archaeology at UCLA.

Whallon, R., \& Lovis, W. (2016). Hunter-Gatherer Landscape Perception and Landscape "Marking" The Multidimensional Construction of Meaning. In W. Lovis \& R. Whallon (Eds.), Marking The Land. HunterGatherer Creation of Meaning in Their Environment (pp. 276-285). Routledge, New York.

Williams, B. J., \& Wobst, H. M. (1974). A Model of Band Society. Memoirs of the Society for American Archaeology, 29, 1-138.

Yesner, D. (1980). Maritime Hunter-Gatherers: Ecology and Prehistory. Current Anthropology, 21, 727-750.

Yesner, D. (1990). Fuegians and Other Hunter-Gatherers of the Sub-Antarctic Region: 'Cultural Devolution' Reconsidered. In B. Meehan \& N. White (Eds.), HunterGatherer Demography. Past and present. Oceania Monograph, 39, 1-22.

Yesner, D. (1996). Island Biogeography and Human Population Persistence in High Latitude Environments: Examples from the Aleutian Islands and Tierra del Fuego. Proceedings of the $10^{\text {th }}$ International Abashiri Symposium (pp. 69-84), Japan.

Yesner, D. (2004). Prehistoric Maritime Adaptations of the Subarctic and Subantarctic Zones: The Aleutian/ Fuegian Connection Reconsidered. Arctic Anthropology, 41(2), 76-97.

Yesner, D. R., Figuerero Torres, M. J., Guichón, R., \& Borrero, L.
A. (2003). Stable Isotope Analysis of Human Bone and Ethnohistoric Subsistence Patterns in Tierra del Fuego. Journal of Anthropological Archaeology, 22, 279-291.

Zamora, E., \& Santana, A. (1979). Características climáticas de la costa occidental de la Patagonia entre las latitudes $46^{\circ} 40^{\prime}$ y $56^{\circ} 30^{\prime} \mathrm{S}$. Anales del Instituto de la Patagonia, 10, 109-144.

Zangrando, A. F. (2009a). Historia evolutiva y subsistencia de cazadores-recolectores marítimos de Tierra del Fuego. Sociedad Argentina de Antropología, Buenos Aires.

Zangrando, A. F. (2009b). Is fishing intensification a direct route to hunter-gatherer complexity? A case study from the Beagle Channel region (Tierra del Fuego, southern South America). World Archaeology, 41(4), 589-608.

Zangrando, A. F., Vázquez, M., \& Tessone, A. (Eds.) (2011). Los cazadores-recolectores del extremo oriental fueguino. Arqueología de península Mitre e Isla de los Estados. Sociedad Argentina de Antropología.

Zangrando, A. F., Borrazzo, K. B., Tivoli, A. M., Alunni, D. V., \& Martinoli, M. P. (2014). El sitio Heshkaia 35: nuevos datos sobre la arqueología de Moat. Revista del Museo de Antropología, 7(1), 11-24.

Zangrando, A. F., Pinto, G., \& Tivoli, A. M. (2016). Decreased foraging return in shellfishing? Species composition and shell size of blue mussel (Mytilus edulis) from a Late Holocene site of the South Coast of Tierra del Fuego. Quaternary International, 427, 160-169.

Zárraga, C. (2016). Cristina Calderón. Memorias de mi abuela Yagán. Ediciones Pix.

Zurro, D., Madella, M., Briz, I., \& Vila, A. (2009). Variability of the phytolith record in fisher-hunter-gatherer sites: An example from the Yamana society (Beagle Channel, Tierra del Fuego, Argentina). Quaternary International, 193, 184-191. 